\title{
On the mean-field theory of the Karlsruhe Dynamo Experiment
}

\author{
K.-H. Rädler, M. Rheinhardt, E. Apstein, and H. Fuchs \\ Astrophysical Institute Potsdam, An der Sternwarte 16, D-15482 Potsdam, Germany
}

Received: 30 August 2001 - Accepted: 30 October 2001

\begin{abstract}
In the Forschungszentrum Karlsruhe an experiment has been constructed which demonstrates a homogeneous dynamo as is expected to exist in the Earth's interior. This experiment is discussed within the framework of meanfield dynamo theory. The main predictions of this theory are explained and compared with the experimental results.
\end{abstract}

Key words. Dynamo, geodynamo, dynamo experiment, mean-field dynamo theory, $\alpha$-effect

\section{Introduction}

It is generally believed that the magnetic fields of the Earth, the Sun and other cosmic bodies result from dynamo mechanisms. In the Forschungszentrum Karlsruhe a device has been constructed for an experiment which should demonstrate a homogeneous dynamo as is expected to exist in the Earth's interior or in cosmic bodies (see, e.g. Stieglitz and Müller, 1996). The experiment was run the first time successfully in December 1999 (see Müller and Stieglitz, 2000, 2002; Stieglitz and Müller, 2001).

The basic idea of this experiment was proposed by Busse (1975, 1978, 1992). It is very similar to an idea discussed before by Gailitis (1967). The essential piece of the experimental device, the dynamo module, is a cylindrical container as shown in Fig. 1, with both radius and height somewhat less than $1 \mathrm{~m}$, through which liquid sodium is driven by external pumps. By means of a system of channels with conducting walls, constituting 52 "spin-generators", helical motions are organized. The flow pattern resembles one considered in the theoretical work of Roberts (1972) which proved to be capable of dynamo action. It is sketched in Fig. 2.

It seems appropriate to discuss the experiment in the framework of the mean-field dynamo theory. Going beyond simple considerations of this kind (Busse, 1992; Busse et al., 1996, 1998; Stieglitz and Müller, 1996) a systematic theory has been developed with mean fields defined by averaging

Correspondence to: K.-H. Rädler (khraedler@aip.de) over areas in planes perpendicular to the cylinder axis covering the cross-sections of several cells (Rädler et al., 1996, 1997a,b, 1998b). The essential induction effect of the fluid motion is then, with respect to the mean magnetic field, described as an anisotropic $\alpha$-effect.

In order to obtain estimates of the self-excitation condition for the magnetic field in the experimental device and to give predictions of its geometrical structure, several kinematic mean-field dynamo models have been investigated, and calculations of the $\alpha$-coefficient and related quantities have been carried out (Rädler et al., 1996, 1997a,b, 1998b, 1999; Rädler and Brandenburg, 2002). In addition, the back-reaction of the magnetic field on the motion has been taken into account in some approximation and so estimates for the saturation field strengths of the dynamo were derived (Rädler et al., 1998a, $2000 a, b)$. Parallel to the elaboration of the mean-field approach to the theory of the experiment several direct numerical simulations of the dynamo process have been carried out (Tilgner, 1996, 1997).

In this paper we give a summarizing representation of the mean-field theory of the experiment and compare the results with the measured data.

\section{The mean-field concept}

Let us assume that the magnetic flux density $\boldsymbol{B}$ inside the dynamo module is governed by the induction equation

$\nabla \times(\eta \nabla \times \boldsymbol{B}-\boldsymbol{u} \times \boldsymbol{B})+\partial_{t} \boldsymbol{B}=\mathbf{0}, \quad \nabla \cdot \boldsymbol{B}=0$,

where $\eta$ is the magnetic diffusivity of the conducting fluid and $\boldsymbol{u}$ the velocity of its motion. The fluid is considered as incompressible, that is $\nabla \cdot \boldsymbol{u}=0$.

We use a Cartesian co-ordinate system $x, y, z$ as indicated in Fig. 1, with the $z$-axis aligned with the cylinder axis but $z=0$ in the middle of the dynamo module. The flow pattern inside the module is assumed to coincide, apart from some boundary layer, with a pattern as depicted in Fig. 2, showing periodicity in $x$ and $y$ with a period length $2 a$, and being independent of $z$. 


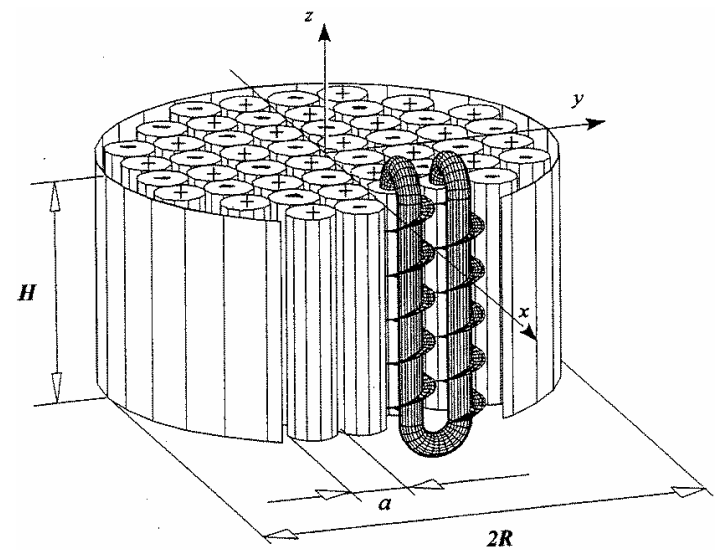

Fig. 1. The dynamo module (after Stieglitz and Müller (1996)). The signs + and - indicate that the fluid moves up or down, respectively, in a given spin generator. $R=0.85 \mathrm{~m}, H=0.71 \mathrm{~m}$, $a=0.21 \mathrm{~m}$.

For the sake of simplicity we ignore until further notice (Sect. 7.1.2) the peculiarities at the curved boundaries of the dynamo module, that is, assume a flow pattern as in Fig. 2 for all $x$ and $y$. We admit, however, at first a dependence of the flow on $z$. When speaking of a "cell" of this pattern we mean a unit like that defined by $0 \leq x, y \leq a$. We further assume, again for simplicity, that $\eta$ does not depend on $x$ and $y$.

Let us follow the lines of the mean-field dynamo theory (see, e.g. Krause and Rädler, 1980). For each given field $F$ we define a mean field $\bar{F}$ by taking an average over an area corresponding to the cross-section of four cells in the $x y$ plane,

$\bar{F}(x, y, z)=\frac{1}{4 a^{2}} \int_{-a}^{a} \int_{-a}^{a} F(x+\xi, y+\eta, z) d \xi d \eta$.

We note that the applicability of the Reynolds averaging rules, which we use in the following, requires that $\bar{F}$ varies only weakly over distances $a$ in $x$ - or $y$-direction. By the way, all what follows applies also with a definition of $\bar{F}$ by averaging over an area corresponding to two cells only (Plunian and Rädler, 2002), but we do not want to consider this possibility here in detail.

We split the magnetic flux density $\boldsymbol{B}$ and the fluid velocity $\boldsymbol{u}$ into mean fields $\overline{\boldsymbol{B}}$ and $\overline{\boldsymbol{u}}$ and remaining fields $\boldsymbol{B}^{\prime}$ and $\boldsymbol{u}^{\prime}$, that is

$\boldsymbol{B}=\overline{\boldsymbol{B}}+\boldsymbol{B}^{\prime}, \quad \boldsymbol{u}=\overline{\boldsymbol{u}}+\boldsymbol{u}^{\prime}$.

Although in this paper $\boldsymbol{B}^{\prime}$ and $\boldsymbol{u}^{\prime}$ are more or less regular fields we will adopt the notation of mean-field theory and call them "fluctuations". As long as we, in the sense explained above, do not consider the situation near the curved boundaries we have $\overline{\boldsymbol{u}}=\mathbf{0}$, that is, $\boldsymbol{u}=\boldsymbol{u}^{\prime}$.

Taking the average of Eq. (1) we see that $\overline{\boldsymbol{B}}$ has to obey

$\nabla \times(\eta \nabla \times \overline{\boldsymbol{B}}-\mathcal{E})+\partial_{t} \overline{\boldsymbol{B}}=\mathbf{0}, \quad \nabla \cdot \overline{\boldsymbol{B}}=0$,

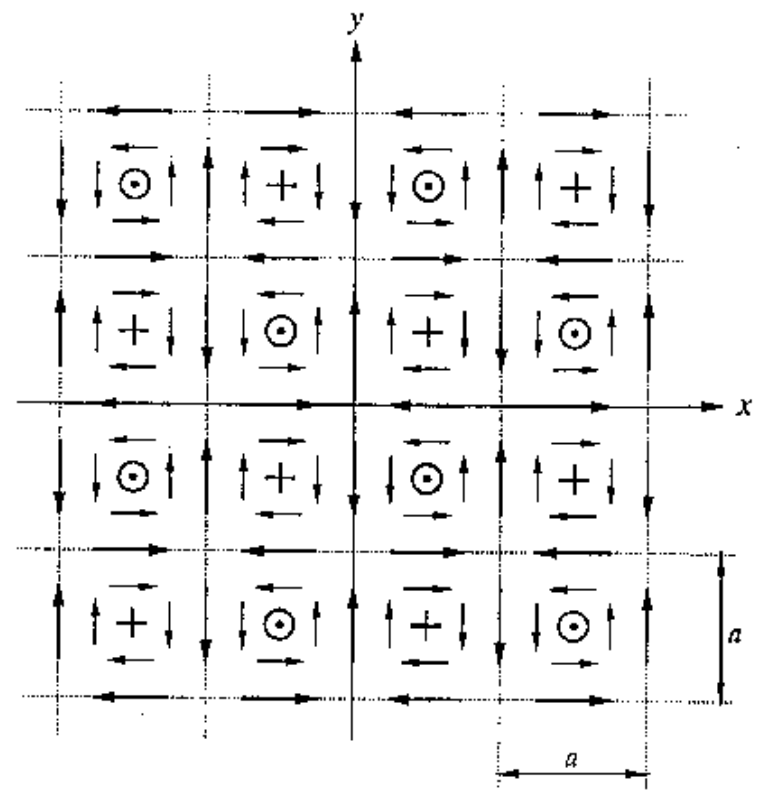

Fig. 2. The Roberts flow pattern. The flow directions correspond to the situation in the dynamo module if the co-ordinate system coincides with that in Fig. 1.

where $\mathcal{E}$, defined by

$\mathcal{E}=\overline{\boldsymbol{u} \times \boldsymbol{B}^{\prime}}$,

is a mean electromotive force due to the fluctuations $\boldsymbol{u}$ and $\boldsymbol{B}^{\prime}$.

The determination of $\mathcal{E}$ for a given $\boldsymbol{u}$ requires the knowledge of $\boldsymbol{B}^{\prime}$. Combining Eqs. (1) and (4) we easily arrive at

$$
\begin{aligned}
& \nabla \times\left(\eta \nabla \times \boldsymbol{B}^{\prime}-\boldsymbol{u} \times \overline{\boldsymbol{B}}-\left(\boldsymbol{u} \times \boldsymbol{B}^{\prime}\right)^{\prime}\right)+\partial_{t} \boldsymbol{B}^{\prime}=\mathbf{0}, \\
& \nabla \cdot \boldsymbol{B}^{\prime}=0,
\end{aligned}
$$

where $\left(\boldsymbol{u} \times \boldsymbol{B}^{\prime}\right)^{\prime}=\boldsymbol{u} \times \boldsymbol{B}^{\prime}-\overline{\boldsymbol{u} \times \boldsymbol{B}^{\prime}}$. We conclude from this that $\boldsymbol{B}^{\prime}$ is, apart from initial and boundary conditions, determined by $\boldsymbol{u}$ and $\overline{\boldsymbol{B}}$ and is linear in $\overline{\boldsymbol{B}}$. We assume here that $\boldsymbol{B}^{\prime}$ vanishes if $\overline{\boldsymbol{B}}$ does so. Thus $\mathcal{E}$, too, can be understood as a quantity determined by $\boldsymbol{u}$ and $\overline{\boldsymbol{B}}$ only and being linear and homogeneous in $\overline{\boldsymbol{B}}$. Of course, $\mathcal{E}$ at a given point in space and time depends not simply on $\boldsymbol{u}$ and $\overline{\boldsymbol{B}}$ in this point but also on their behaviour in the neighbourhood of this point.

We adopt the assumption that $\overline{\boldsymbol{B}}$ varies only weakly in space and time so that $\overline{\boldsymbol{B}}$ and its first spatial derivatives in this point are sufficient to define the behaviour of $\overline{\boldsymbol{B}}$ in the relevant surroundings. Then $\mathcal{E}$ can be represented in the form

$\mathcal{E}=a_{i j} \bar{B}_{j}+b_{i j k} \frac{\partial \bar{B}_{j}}{\partial x_{k}}$,

where the tensors $a_{i j}$ and $b_{i j k}$ are averaged quantities determined by $\boldsymbol{u}$. We use here and in the following the notation $x_{1}=x, x_{2}=y, x_{3}=z$ and adopt the summation convention. Of course, the neglect of contributions to $\mathcal{E}$ with higher-order spatial derivatives or with time derivatives of $\overline{\boldsymbol{B}}$ remains to be checked in all applications (see Sect. 7.3). 
Before giving results of calculations of $\mathcal{E}$ with specific assumptions on $\boldsymbol{u}$, we write down its most general form compatible with Eq. (7), which can be determined by standard methods of mean-field theory (see, e.g. Krause and Rädler, 1980). Due to our definition of averages and the periodicity of the flow pattern, $a_{i j}$ and $b_{i j k}$ are independent of $x$ and $y$. Clearly a $90^{\circ}$ rotation of the flow pattern about the $z$-axis as well as a shift by the length $a$ along the $x$-or the $y$-axis change only the sign of $\boldsymbol{u}$ so that simultaneous rotation and shift leave $\boldsymbol{u}$ unchanged. This is sufficient to conclude that $a_{i j}$ and $b_{i j k}$ are axisymmetric tensors with respect to the $z$ axis. That is, $a_{i j}$ is a linear combination of $\delta_{i j}, \varepsilon_{i j l} e_{l}$ and $e_{i} e_{j}$, and $b_{i j k}$ a linear combination of $\varepsilon_{i j k}, \delta_{i j} e_{k}, \delta_{i k} e_{j}, \delta_{j k} e_{i}$, $\varepsilon_{i j l} e_{l} e_{k}, \varepsilon_{i k l} e_{l} e_{j}, \varepsilon_{j k l} e_{l} e_{i}$ and $e_{i} e_{j} e_{k}$. Here $\delta_{i j}$ means the Kronecker tensor, $\varepsilon_{i j k}$ the Levi-Civita tensor and $\boldsymbol{e}$ the unit vector in $z$-direction. With this specification of $a_{i j}$ and $b_{i j k}$ Eq. (7) turns into

$$
\begin{aligned}
\mathcal{E}= & -\alpha_{\perp} \overline{\boldsymbol{B}}-\left(\alpha_{\|}-\alpha_{\perp}\right)(\boldsymbol{e} \cdot \overline{\boldsymbol{B}}) \boldsymbol{e}-\gamma \boldsymbol{e} \times \overline{\boldsymbol{B}} \\
& -\beta_{\perp} \nabla \times \overline{\boldsymbol{B}}-\left(\beta_{\|}-\beta_{\perp}\right)(\boldsymbol{e} \cdot(\nabla \times \overline{\boldsymbol{B}})) \boldsymbol{e} \\
& -\beta_{3} \boldsymbol{e} \times(\nabla(\boldsymbol{e} \cdot \overline{\boldsymbol{B}})+(\boldsymbol{e} \cdot \nabla) \overline{\boldsymbol{B}}) \\
& -\delta_{1} \nabla(\boldsymbol{e} \cdot \overline{\boldsymbol{B}})-\delta_{2}(\boldsymbol{e} \cdot \nabla) \overline{\boldsymbol{B}}-\delta_{3}(\boldsymbol{e} \cdot \nabla(\boldsymbol{e} \cdot \overline{\boldsymbol{B}})) \boldsymbol{e},
\end{aligned}
$$

with coefficients $\alpha_{\perp}, \alpha_{\|}, \gamma, \beta_{\perp}, \beta_{\|}, \ldots$, which are averaged quantities determined by $\boldsymbol{u}$ and are independent of $x$ and $y$ but may depend on $z$. The terms with $\alpha_{\perp}$ and $\alpha_{\|}$describe the $\alpha$-effect, which is in general anisotropic, those with $\beta_{\perp}$ and $\beta_{\|}$give rise to the introduction of a mean-field conductivity different from the original electric conductivity of the fluid and again in general anisotropic. The term with $\gamma$ describes a transport of mean magnetic flux like that due to a fluid motion with the velocity $-\gamma \boldsymbol{e}$. The remaining terms are less easily to interpret. We note that in contrast to the $\beta_{\perp}$ and $\beta_{\|}$ terms the $\beta_{3}$ term is not connected with $\nabla \times \overline{\boldsymbol{B}}$ but with the symmetric part of the gradient tensor of $\overline{\boldsymbol{B}}$ and can therefore not be interpreted in the sense of a mean-field conductivity.

We proceed now to the case in which $\boldsymbol{u}$ is independent of $z$ (but return to the case in which it depends on $z$ in Sect. 7.1.1). Consider for a moment $\overline{\boldsymbol{B}}$ as a homogeneous field in the $z$ direction. Then $\nabla \times(\boldsymbol{u} \times \overline{\boldsymbol{B}})$ vanishes, and we have to conclude from Eq. (6) that $\boldsymbol{B}^{\prime}=\mathbf{0}$. This in turn leads to $\mathcal{E}=\mathbf{0}$, and therefore Eq. (8) can only be correct if $\alpha_{\|}=0$. Returning again to arbitrary $\overline{\boldsymbol{B}}$ we further consider the fact that averaged quantities determined by $\boldsymbol{u}$ can never imply a possibility to distinguish between the positive and the negative $z$-directions. This means that $\mathcal{E}$ in the form given by Eq. (8) must be invariant under exchanging $\boldsymbol{e}$ with $-\boldsymbol{e}$, which requires that $\gamma=\delta_{1}=\delta_{2}=\delta_{3}=0$. Thus we arrive at

$$
\begin{aligned}
\mathcal{E}= & -\alpha_{\perp}(\overline{\boldsymbol{B}}-(\boldsymbol{e} \cdot \overline{\boldsymbol{B}}) \boldsymbol{e}) \\
& -\beta_{\perp} \nabla \times \overline{\boldsymbol{B}}-\left(\beta_{\|}-\beta_{\perp}\right)(\boldsymbol{e} \cdot(\nabla \times \overline{\boldsymbol{B}})) \boldsymbol{e} \\
& -\beta_{3} \boldsymbol{e} \times(\nabla(\boldsymbol{e} \cdot \overline{\boldsymbol{B}})+(\boldsymbol{e} \cdot \nabla) \overline{\boldsymbol{B}}) .
\end{aligned}
$$

Here the $\alpha$-effect has an extremely anisotropic form. It is able to drive electric currents in the $x$ - and $y$-direction but not in the $z$-direction.

\section{Simple kinematic mean-field dynamo models}

Let us consider simple kinematic mean-field dynamo models which reflect essential features of the experimental device. We assume here that the mean magnetic flux density $\overline{\boldsymbol{B}}$ inside a cylindrical body which corresponds to the dynamo module is governed by Eq. (4). For the sake of simplicity we specify the electromotive force $\mathcal{E}$ so that it covers only the anisotropic $\alpha$-effect and consider both $\eta$ and $\alpha_{\perp}$ as independent of space coordinates and time. So we have inside this body

$$
\begin{gathered}
\eta \nabla^{2} \overline{\boldsymbol{B}}-\alpha_{\perp} \nabla \times(\overline{\boldsymbol{B}}-(\boldsymbol{e} \cdot \overline{\boldsymbol{B}}) \boldsymbol{e})-\partial_{t} \overline{\boldsymbol{B}}=\mathbf{0}, \\
\nabla \cdot \overline{\boldsymbol{B}}=0 .
\end{gathered}
$$

In the outer space the $\alpha$-effect is taken to be zero, and various assumptions concerning the electric conductivity are considered, which will be specified later.

In models of that kind several types of magnetic fields showing different symmetries with respect to the axis and the middle plane of the cylinder are possible. Equations (10) allow independent solutions $\overline{\boldsymbol{B}}$ which vary like $\exp (\operatorname{im} \varphi)$ with the azimuthal co-ordinate $\varphi$ of a cylindrical system whose axis coincides with that of the dynamo module. The fields with $m=0$ are symmetric, such with $m \neq 0$ non-symmetric with respect to this axis. In the axisymmetric case there are again two independent types of fields. For one the poloidal part is antisymmetric and the toroidal part symmetric with respect to the middle plane, and for the other vice versa. Such fields are denoted by AS or SA, respectively. The poloidal part of an AS field is dipole-like, that of a SA field quadrupole-like. In the simplest non-axisymmetric case, that is $m=1$, the field corresponds roughly to that of a dipole lying in the middle plane, but its field lines above and below this plane are distorted as it would happen with opposite rotations of the fluid in these regions about the $z$-axis. In the case $m=0$ we have $\bar{B}_{x}=\bar{B}_{y}=0$ on the $z$-axis, in the case $m=1$ we have $\bar{B}_{z}=0$, and for $m \geq 2$ finally $\bar{B}_{x}=\bar{B}_{y}=\bar{B}_{z}=0$.

We may measure all lengths in units of the radius $R$ of the cylindrical body considered and the time in units of $R^{2} / \eta$. Then Eq. (10) takes the form

$$
\begin{gathered}
\nabla^{2} \overline{\boldsymbol{B}}-C \nabla \times(\overline{\boldsymbol{B}}-(\boldsymbol{e} \cdot \overline{\boldsymbol{B}}) \boldsymbol{e})-\partial_{t} \overline{\boldsymbol{B}}=\mathbf{0}, \\
\nabla \cdot \overline{\boldsymbol{B}}=0,
\end{gathered}
$$

where $C$ is a dimensionless measure of the $\alpha$-effect,

$$
C=\frac{\alpha_{\perp} R}{\eta} \text {. }
$$

In general the solutions $\overline{\boldsymbol{B}}$ of Eq. (11) for a given $m$ are superpositions of independent solutions each of which varies with time like $\exp (p t)$, where $p$ is in general complex. For each such solution Eq. (11) together with proper boundary conditions pose an eigenvalue problem with $p$ being the eigenvalue parameter. Of course, the eigenvalues $p$ depend on $C$. Clearly the growth rate $\lambda$, given by $\lambda=\Re(p)$, must be 


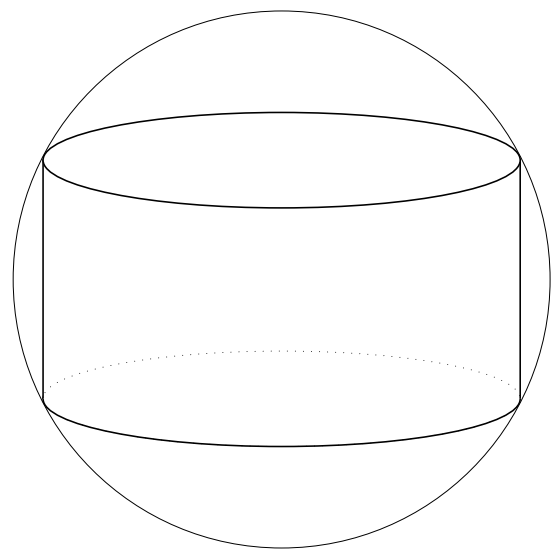

Fig. 3. Concerning the numerical calculations: the cylindrical body embedded in a sphere

negative for small $C$. For each type of solutions with a given $m$, in the case $m=0$ with a given specification AS or SA, we define a marginal value $C^{*}$ of $C$ so that all $\lambda$ are negative for $C<C^{*}$, but at least one of them vanishes at $C=C^{*}$. This marginal value $C^{*}$ defines the self-excitation condition for the corresponding type of magnetic fields.

Estimates for the marginal values $C^{*}$ where derived from models treated in other contexts in which the $\alpha$-effect was not restricted to a finite cylinder but was assumed to act either in all space, in an infinite slab, in an infinite cylinder, or in a sphere. The results obtained in this way suggest that for our cylindrical body $C^{*}<10$ (Rädler et al., 1996; Gailitis, 1967).

Several numerical studies of dynamo models as described above, that is, with the $\alpha$-effect restricted to a finite cylinder, have been carried out. For most of them a code developed for spherical models (Fuchs et al., 1993) was used, with the cylinder embedded in an electrically conducting sphere surrounded by free space. As sketched in Fig. 3 the smallest sphere just containing the cylinder was chosen. The conductivity of the parts of the sphere outside the cylinder was assumed to be equal to $\xi$ times that inside the cylinder. In these calculations the ratio of radius $R$ and height $H$ of the cylinder was fixed at $R / H=1$. Using another method (Dobler and Rädler, 1998), models with the same conductivity everywhere inside and outside the cylinder and $R / H=1.21$ were also investigated. We denote the two kinds of models by (i) and (ii). The marginal values $C^{*}$ for some magnetic fields with low $m$ are presented in Table 1 (see also Rädler et al., 1996, 1998b). Figs. 4 and 5 exhibit examples of field structures. All these fields are steady, that is, non-oscillatory. Our results show that the non-axisymmetric field with $m=1$ is clearly preferred over the axisymmetric and the other nonaxisymmetric fields. That is, magnetic fields of the symmetry type $m=1$ can be generated or maintained with the lowest requirements concerning the $\alpha$-effect.

Results for a more sophisticated mean-field dynamo model will be given later (Sect. 7.4).
Table 1. Marginal values $C^{*}$ for cylindrical dynamo models of types (i) and (ii) and different types of magnetic fields

\begin{tabular}{ccccc}
\hline model & $\begin{array}{c}m=0 \\
\text { AS }\end{array}$ & $\begin{array}{c}m=0 \\
\text { SA }\end{array}$ & $m=1$ & $m=2$ \\
& & & \\
(i) $\xi=1$ & 8.22 & 8.46 & 6.41 & 8.62 \\
(i) $\xi=0.01$ & 8.64 & 9.18 & 7.70 & 9.67 \\
(i) $\xi=0.001$ & 9.02 & 9.60 & 8.12 & 10.12 \\
(ii) & 8.55 & 8.55 & 6.28 & 8.55 \\
\hline
\end{tabular}

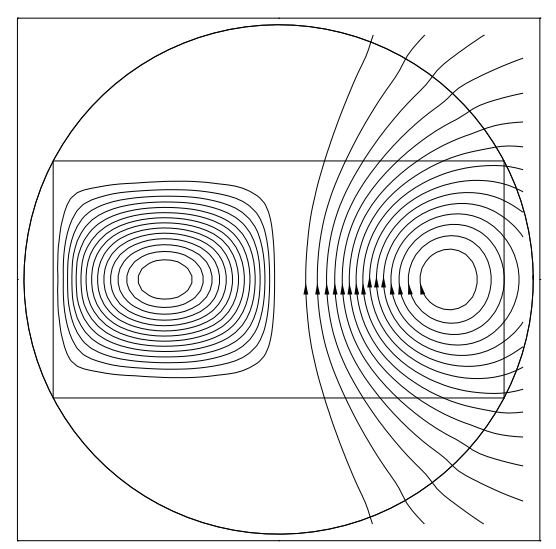

Fig. 4. Magnetic field of type $m=0 \mathrm{AS}, \xi=0.01$. Left: isolines of the toroidal part, right: field lines of the poloidal part

\section{The $\alpha$-effect under idealized conditions}

\subsection{General considerations}

In order to formulate the self-excitation condition in terms of the rates of the flow through the spin generators we need to know how $\alpha_{\perp}$, or $C$, depends on them. In the following we focus attention on the calculation of the coefficient $\alpha_{\perp}$ in the case in which $\boldsymbol{u}$ is independent of $z$ (but will come to a case in which it depends on $z$ in Sect. 7.1.1). For this purpose it is sufficient to restrict our considerations to the case in which $\overline{\boldsymbol{B}}$ is a homogeneous field. For the sake of simplicity we further assume again that $\eta$ is constant. Then $\overline{\boldsymbol{u} \times \boldsymbol{B}^{\prime}}$ is also constant, that is $\left.\nabla \times \overline{\left(\bar{u} \times \boldsymbol{B}^{\prime}\right.}\right)=\mathbf{0}$, and Eq. (6) for $\boldsymbol{B}^{\prime}$ takes the simple form

$$
\begin{gathered}
\eta \nabla^{2} \boldsymbol{B}^{\prime}+\left(\boldsymbol{B}^{\prime} \cdot \nabla\right) \boldsymbol{u}-(\boldsymbol{u} \cdot \nabla) \boldsymbol{B}^{\prime}-\partial_{t} \boldsymbol{B}^{\prime}=-(\overline{\boldsymbol{B}} \cdot \nabla) \boldsymbol{u}, \\
\nabla \cdot \boldsymbol{B}^{\prime}=0 .
\end{gathered}
$$

We may assume that $\boldsymbol{B}^{\prime}$ like $\overline{\boldsymbol{B}}$ is independent of $z$. Let us put $\boldsymbol{B}^{\prime}=\boldsymbol{B}_{\perp}^{\prime}+\boldsymbol{B}_{\|}^{\prime}$ and $\boldsymbol{u}=\boldsymbol{u}_{\perp}+\boldsymbol{u}_{\|}$with $\boldsymbol{B}_{\perp}^{\prime}=\boldsymbol{B}^{\prime}-\left(\boldsymbol{e} \cdot \boldsymbol{B}^{\prime}\right) \boldsymbol{e}$ and $\boldsymbol{B}_{\|}^{\prime}=\left(\boldsymbol{e} \cdot \boldsymbol{B}^{\prime}\right) \boldsymbol{e}$, and $\boldsymbol{u}_{\perp}$ and $\boldsymbol{u}_{\|}$defined analogously. We put further $\boldsymbol{u}_{\perp}=u_{\perp} \tilde{\boldsymbol{u}}_{\perp}$ and $\boldsymbol{u}_{\|}=u_{\|} \tilde{\boldsymbol{u}}_{\|}$, where $u_{\perp}$ and $u_{\|}$are factors independent of $x$ and $y$ characterizing the magnitudes of $\boldsymbol{u}_{\perp}$ and $\boldsymbol{u}_{\|}$, and $\tilde{\boldsymbol{u}}_{\perp}$ and $\tilde{\boldsymbol{u}}_{\|}$fields which are normalized in some way. We may conclude from Eq. (13) that $\boldsymbol{B}_{\perp}^{\prime}$ depends only on $u_{\perp}$ and not on $u_{\|}$, and that $\boldsymbol{B}_{\|}^{\prime}$ depends again on $u_{\perp}$ but only in a linear and homogeneous way on $u_{\|}$. This 

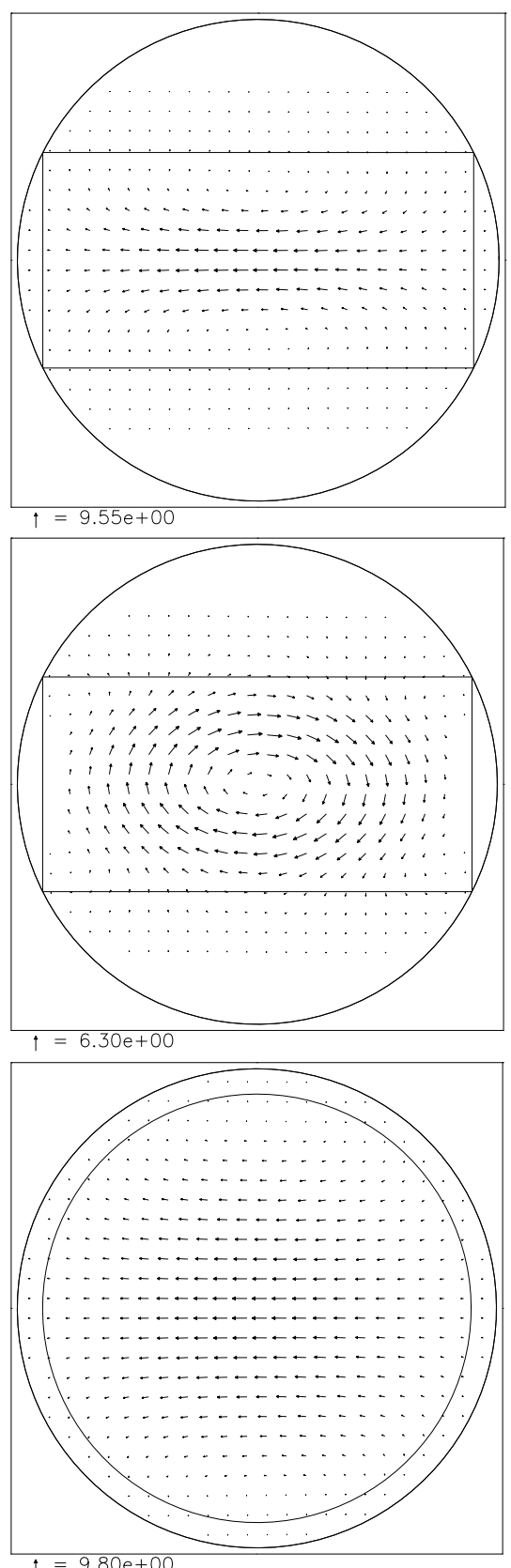

Fig. 5. Magnetic field of type $m=1, \xi=0.01$. Top: plane $y=0$, middle: plane $x=0$, bottom: plane $z=0$

implies that $\mathcal{E}$ and therefore $\alpha_{\perp}$ may depend in a complex way on $u_{\perp}$ but must be linear and homogeneous in $u_{\|}$.

We will consider here two kinds of flow patterns, a highly idealized one previously investigated by Roberts (1972) and another one which is more realistic in view of the spin generators of the experimental device. As indicated in Fig. 1 each spin generator consists of an central axial and an outer helical channel. In the ideal situation the fluid outside the channels is at rest.

For sufficiently small magnitudes of $\boldsymbol{u}$ the so-called second-order approximation can be justified, which consists in the neglect of the two terms with $\boldsymbol{u}$ on the left-hand side of Eq. (13). In both cases we will start with this simple approximation but then proceed to results for arbitrary magnitudes of $\boldsymbol{u}$.

\subsection{Roberts flow}

We define the Roberts flow by

$\boldsymbol{u}=u_{\perp} \frac{a}{2} \boldsymbol{e} \times \nabla \chi-u_{\|}\left(\frac{\pi}{2}\right)^{2} \chi \boldsymbol{e}$,

$\chi=\sin \left(\frac{\pi}{a} x\right) \sin \left(\frac{\pi}{a} y\right)$,

or, more explicitly,

$u_{x}=-u_{\perp} \frac{\pi}{2} \sin \left(\frac{\pi}{a} x\right) \cos \left(\frac{\pi}{a} y\right)$,

$u_{y}=u_{\perp} \frac{\pi}{2} \cos \left(\frac{\pi}{a} x\right) \sin \left(\frac{\pi}{a} y\right)$,

$u_{z}=-u_{\|}\left(\frac{\pi}{2}\right)^{2} \sin \left(\frac{\pi}{a} x\right) \sin \left(\frac{\pi}{a} y\right)$.

Here $u_{\perp}$ is the average of the modulus of the velocity component in the $x y$-plane perpendicular to a line running from the centre of a cell to its boundary taken over this line, e.g., the average of $-u_{x}$ at $x=a / 2$ over $0 \leq y \leq a / 2$, or of $u_{y}$ at $y=a / 2$ over $0 \leq x \leq a / 2$, and $u_{\|}$is the average of the modulus of $u_{z}$ over the cross-section of a cell, e.g. $0 \leq x, y \leq a$, that is,

$u_{\perp}=-\frac{2}{a} \int_{0}^{a / 2} u_{x}(a / 2, y) d y$,

$u_{\|}=-\frac{1}{a^{2}} \int_{0}^{a} \int_{0}^{a} u_{z}(x, y) d x d y$.

Using $u_{\perp}$ and $u_{\|}$we define magnetic Reynolds numbers $R m_{\perp}$ and $R m_{\|}$by

$R m_{\perp}=\frac{u_{\perp} a}{2 \eta}, \quad R m_{\|}=\frac{u_{\|} a}{\eta}$.

We also introduce volumetric flow rates $V_{\perp}$ and $V_{\|}$by

$V_{\perp}=\frac{a h}{2} u_{\perp}, \quad V_{\|}=a^{2} u_{\|}$,

where $h$ means a length characterizing the pitches of the stream lines, which we will later identify with the pitch of the helical channel of a spin generator. We note that $V_{\perp}=R m_{\perp} h \eta$ and $V_{\|}=R m_{\|} a \eta$.

We consider first the second-order approximation, which applies in the limit of small $\boldsymbol{u}$, more precisely for $R m_{\perp}, R m_{\|} \ll 1$. It allows us a simple determination of the steady solution $\boldsymbol{B}^{\prime}$ of Eq. (13) (see Appendix A). Calculating then $\overline{\boldsymbol{u} \times \boldsymbol{B}^{\prime}}$ we find

$\alpha_{\perp}=\frac{\pi^{2}}{32} \frac{a}{\eta} u_{\perp} u_{\|}=\frac{\pi^{2}}{16} \frac{\eta}{a} R m_{\perp} R m_{\|}=\frac{\pi^{2}}{16} \frac{V_{\perp} V_{\|}}{a^{2} h \eta}$. 


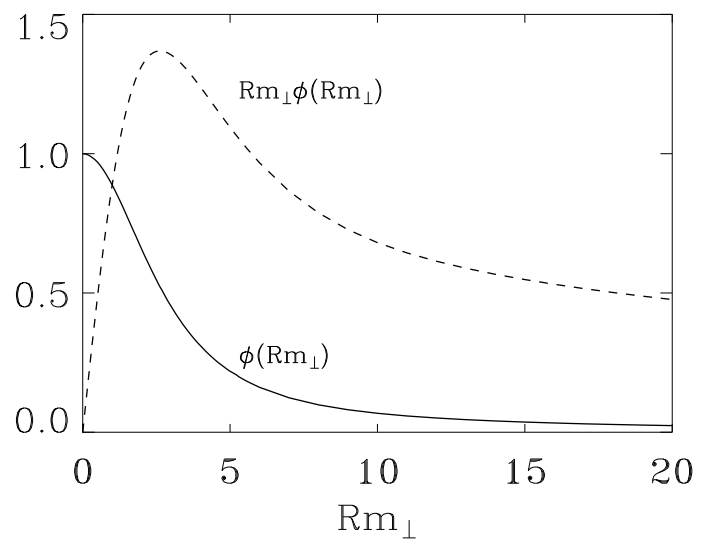

Fig. 6. The functions $\phi\left(R m_{\perp}\right)$ and $R m_{\perp} \phi\left(R m_{\perp}\right)$

According to our remarks in Sect. 4.1 the Relations (19) must also hold true for arbitrary $u_{\|}$, that is, arbitrary $R m_{\|}$ and $V_{\|}$. Therefore, generalizations of the form

$\alpha_{\perp}=\frac{\pi^{2}}{32} \frac{a}{\eta} u_{\perp} u_{\|} \phi\left(u_{\perp} a / 2 \eta\right)$

$=\frac{\pi^{2}}{16} \frac{\eta}{a} R m_{\perp} R m_{\|} \phi\left(R m_{\perp}\right)$

$=\frac{\pi^{2}}{16} \frac{V_{\perp} V_{\|}}{a^{2} h \eta} \phi\left(V_{\perp} / h \eta\right)$

must apply for arbitrary $u_{\perp}$ and $u_{\|}$, or arbitrary $R m_{\perp}, R m_{\|}$, $V_{\perp}$ and $V_{\|}$. Here $\phi$ is a function satisfying $\phi(0)=1$, which remains to be determined.

Equations (13) for $\boldsymbol{B}^{\prime}$ have been reduced to a system of ordinary differential equations for its Fourier components with respect to $x$ and $y$, and these have been integrated numerically. From the result for $\boldsymbol{B}^{\prime}$ in the steady final state again $\overline{\boldsymbol{u} \times \boldsymbol{B}^{\prime}}$ and in the end $\alpha_{\perp}$ have been calculated (see Rädler et al., 1997a, b). In this way the function $\phi\left(R m_{\perp}\right)$ shown in Fig. 6 was determined. As can also be seen there $\alpha_{\perp}$, considered as function of $R m_{\perp}$, first grows with growing $R m_{\perp}$, reaches a maximum at $R m_{\perp}=2.6$, and then decays again. This decay results from magnetic flux expulsion out of the rotating inner parts of each cell. By the way, in agreement with results of asymptotic studies (Soward, 1987) it was found that $\phi$ behaves like $R m_{\perp}^{-3 / 2}$ and, therefore, $\alpha_{\perp}$ like $R m_{\perp}^{-1 / 2}$ as $R m_{\perp} \rightarrow \infty$.

Let us interpret our result in view of an array of spin generators. We denote the volumetric flow rates through the central and the helical channel of a spin generator by $V_{\mathrm{C}}$ and $V_{\mathrm{H}}$, respectively. Clearly, $V_{\perp}$ corresponds to $V_{\mathrm{H}}$, and $V_{\|}$to $V_{\mathrm{C}}+V_{\mathrm{H}}$. Then, using Eq. (20) and putting $V_{\perp}=V_{\mathrm{H}}$ and $V_{\|}=V_{\mathrm{C}}+V_{\mathrm{H}}$, we arrive at

$\alpha_{\perp}=\frac{\pi^{2}}{16} \frac{V_{\mathrm{H}}}{a^{2} h \eta}\left(V_{\mathrm{C}}+V_{\mathrm{H}}\right) \phi\left(V_{\mathrm{H}} / h \eta\right)$.

Figure 7 shows the dependence of $a^{2} \alpha_{\perp}$ on $V_{\mathrm{C}}$ and $V_{\mathrm{H}}$ for $h=0.905 a$ (chosen with a view to Eq. 32 ). There is a

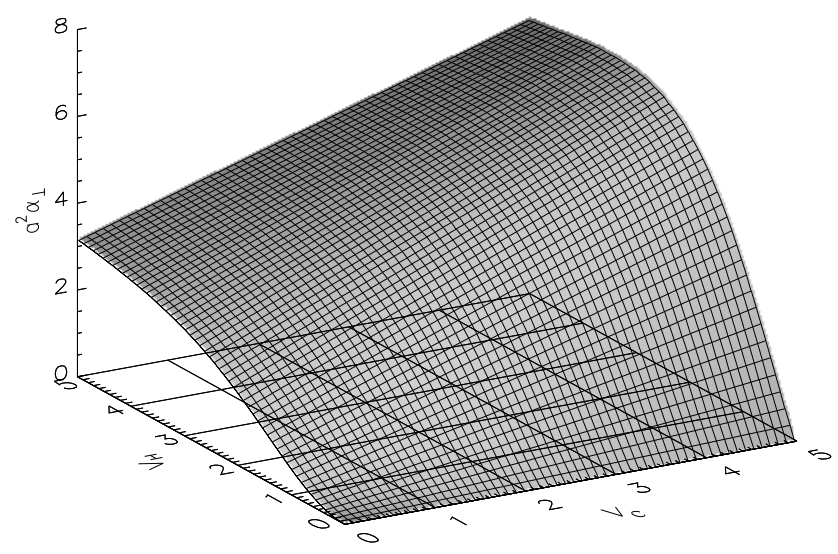

Fig. 7. The dependence of $a^{2} \alpha_{\perp}$ on $V_{\mathrm{C}}$ and $V_{\mathrm{H}}$, all three quantities measured in units of $a \eta$, for $h=0.905 a$

value $V_{\mathrm{C}}^{*}$ (in the units of Fig. 7 we have $V_{\mathrm{C}}^{*}<2.5$ ) so that $\alpha_{\perp}\left(V_{\mathrm{C}}, V_{\mathrm{H}}\right)$ for any fixed $V_{\mathrm{C}}<V_{\mathrm{C}}^{*}$ grows with $V_{\mathrm{H}}$. For $V_{\mathrm{C}}>V_{\mathrm{C}}^{*}$, however, $\alpha_{\perp}\left(V_{\mathrm{C}}, V_{\mathrm{H}}\right.$ grows for small $V_{\mathrm{H}}$ only, then reaches a maximum and decays again for larger $V_{\mathrm{H}}$. This decay is again a consequence of the magnetic flux expulsion from the inner parts of the cells. Later in Fig. 11 isolines of $C$ in the $V_{\mathrm{C}} V_{\mathrm{H}}$-plane are shown, which because of $C=\alpha_{\perp} R / \eta$ can easily be interpreted as isolines of $\alpha_{\perp}$.

\subsection{Spin generator flow}

Let us now proceed to a flow pattern which is more realistic in view of the flow in the array of spin generators in the experimental device. In order to describe the fluid velocity $\boldsymbol{u}$ we consider it at first only in a single cell, say $0 \leq x, y \leq a$. We introduce there a cylindrical co-ordinate system $\varrho, \varphi$, $z$ with the axis $\varrho=0$ at the centre of this cell, that is, at $x=y=a / 2$. In this cell the fluid velocity $\boldsymbol{u}$ is, with respect to this co-ordinate system, assumed to be given by

$u_{\varrho}=0, \quad u_{\varphi}=u_{\varphi}(\varrho), \quad u_{z}=u_{z}(\varrho)$,

with $u_{\varphi}$ and $u_{z}$ depending on $\varrho$ only and vanishing for $\varrho>a / 2$. The complete flow pattern in all $x y$-plane is then defined by periodic continuation of the pattern described for the cell considered here to all cells, with changes of the sign from each cell to its neighbouring cells as indicated in Fig. 2.

We specify $\boldsymbol{u}$ further by putting

$u_{\varphi}=0, u_{z}=-u(\varrho)$ for $0<\varrho \leq \varrho_{1}$

$u_{\varphi}=-\omega(\varrho) \varrho, u_{z}=-\frac{h}{2 \pi} \omega(\varrho)$ for $\varrho_{1}<\varrho \leq \varrho_{2}$

$u_{\varphi}=0, u_{z}=0$ for $\varrho>\varrho_{2}$.

Here $u$ and $\omega$ are arbitrary functions of $\varrho$. Further $\varrho_{1}$ and $\varrho_{2}$ mean the radius of the central channel and the outer radius of the helical one, respectively, and $h$ the pitch of the helical channel; see Fig. 8. The - signs in Eq. (23) make that $u$ and $\omega$ can be considered as positive. The coupling between $u_{\varphi}$ 


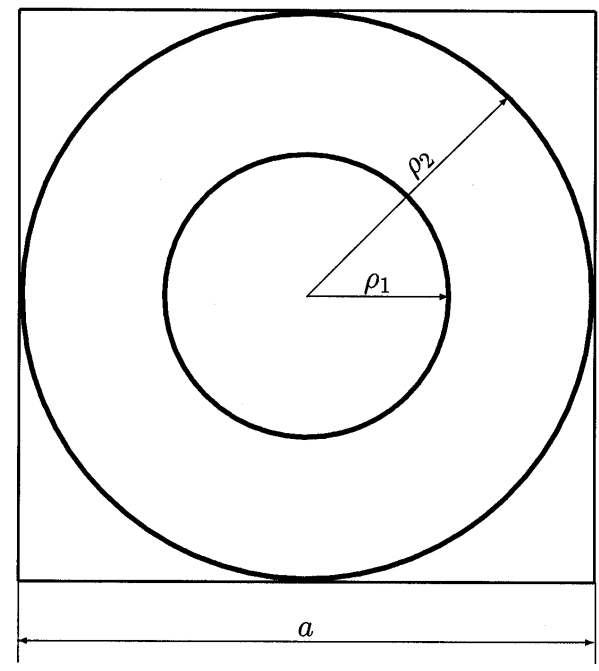

Fig. 8. Cross-section of a spin generator

and $u_{z}$ in $\varrho_{1}<\varrho \leq \varrho_{2}$ considers the constraint on the flow resulting from those walls of the helical channel which are no cylindrical surfaces. We define further the averages $u_{\perp}$, $u_{\| \mathrm{C}}$ and $u_{\| \mathrm{H}}$ of the relevant velocities,

$u_{\perp}=\frac{2}{a} \int_{\varrho_{1}}^{\varrho_{2}} \omega(\varrho) \varrho d \varrho$

$u_{\| \mathrm{C}}=\frac{2 \pi}{a^{2}} \int_{0}^{\varrho_{1}} u(\varrho) \varrho d \varrho, u_{\| \mathrm{H}}=\frac{h}{a^{2}} \int_{\varrho_{1}}^{\varrho_{2}} \omega(\varrho) \varrho d \varrho$,

and note that $u_{\| \mathrm{H}}=(h / 2 a) u_{\perp}$. On this basis we define magnetic Reynolds numbers $R m_{\perp}, R m_{\| \mathrm{C}}$ and $R m_{\| \mathrm{H}}$ by

$R m_{\perp}=\frac{u_{\perp} a}{2 \eta}, \quad R m_{\| \mathrm{C}}=\frac{u_{\| \mathrm{C}} a}{\eta}, \quad R m_{\| \mathrm{H}}=\frac{u_{\| \mathrm{H}} a}{\eta}$,

where, of course, $R m_{\| \mathrm{H}}=(h / a) R m_{\perp}$. Note that the averages $u_{\perp}, u_{\| \mathrm{C}}$ and $u_{\| \mathrm{H}}$ are related to the length $a / 2$ or the area $a^{2}$ and not to the actual extents of the respective flows. By this reason also $R m_{\perp}, R m_{\| \mathrm{C}}$ and $R m_{\| \mathrm{H}}$ have to be interpreted with some care. Finally we introduce the volumetric flow rates $V_{\mathrm{C}}$ and $V_{\mathrm{H}}$ through the central and the helical channel,

$V_{\mathrm{C}}=a^{2} u_{\| \mathrm{C}}, \quad V_{\mathrm{H}}=\frac{a h}{2} u_{\perp}=a^{2} u_{\| \mathrm{H}}$.

In the second-order approximation, that is for sufficiently small magnitudes of $\boldsymbol{u}$, more precisely for $R m_{\perp}, R m_{\| \mathrm{C}}, R m_{\| \mathrm{H}} \ll 1$, the quantity $\alpha_{\perp}$ can be calculated by taking the average of, say, $\left(\boldsymbol{u} \times \boldsymbol{B}^{\prime}\right)_{x}$ over a single cell ignoring the contributions to $\boldsymbol{B}^{\prime}$ resulting from the flow outside, and dividing it by $\bar{B}_{x}$. It can be shown that these contributions to $\boldsymbol{B}^{\prime}$ produce only such parts of $\left(\boldsymbol{u} \times \boldsymbol{B}^{\prime}\right)_{x}$ which vanish under averaging (see Appendix B). Assuming then that $\boldsymbol{u}$ in the considered cell is given by Eq. (22) and vanishes outside, it is again easy to find the steady solution of
Eq. (13) (see Appendix A). So we arrive at

$$
\begin{gathered}
\alpha_{\perp}=\frac{\pi}{a^{2} \eta} \int_{0}^{\varrho_{2}}\left(u_{\varphi}(\varrho) \int_{0}^{\varrho} u_{z}\left(\varrho^{\prime}\right) \varrho^{\prime} d \varrho^{\prime}\right. \\
\left.+u_{z}(\varrho) \varrho \int_{\varrho}^{\varrho_{2}} u_{\varphi}\left(\varrho^{\prime}\right) d \varrho^{\prime}\right) d \varrho .
\end{gathered}
$$

Interestingly enough, as can be shown by an integration by parts, the two double integrals on the right-hand side are equal to each other. We may therefore also write

$$
\begin{aligned}
\alpha_{\perp} & =\frac{2 \pi}{a^{2} \eta} \int_{0}^{\varrho_{2}}\left(u_{\varphi}(\varrho) \int_{0}^{\varrho} u_{z}\left(\varrho^{\prime}\right) \varrho^{\prime} d \varrho^{\prime}\right) d \varrho \\
& =\frac{2 \pi}{a^{2} \eta} \int_{0}^{\varrho_{2}}\left(u_{z}(\varrho) \varrho \int_{\varrho}^{\varrho_{2}} u_{\varphi}\left(\varrho^{\prime}\right) d \varrho^{\prime}\right) d \varrho .
\end{aligned}
$$

Let us evaluate these relations for $\alpha_{\perp}$ with the more specific assumptions (Eq. 23) on $u_{\varphi}$ and $u_{z}$. We find then

$$
\begin{aligned}
\alpha_{\perp} & =\frac{a}{2 \eta} u_{\perp}\left(u_{\| \mathrm{C}}+\frac{1}{2} u_{\| \mathrm{H}}\right) \\
& =\frac{\eta}{a} R m_{\perp}\left(R m_{\| \mathrm{C}}+\frac{1}{2} R m_{\| \mathrm{H}}\right) \\
& =\frac{V_{\mathrm{H}}}{a^{2} h \eta}\left(V_{\mathrm{C}}+\frac{1}{2} V_{\mathrm{H}}\right) .
\end{aligned}
$$

Obviously the axial flow in the central channel of the spin generator, where no azimuthal flow exists, is more effective in view of $\alpha_{\perp}$ than the axial component of the flow in the helical channel. Note that in particular the relation between $\alpha_{\perp}, V_{\mathrm{C}}$ and $V_{\mathrm{H}}$ applies independently of $\varrho_{1}$ and $\varrho_{2}$.

We leave now the second-order approximation. With the same arguments as used in the case of the Roberts flow we find that the general forms of $\alpha_{\perp}$, which apply for arbitrary $u_{\perp}, u_{\| \mathrm{C}}, u_{\| \mathrm{H}}, R m_{\perp}, \cdots V_{\mathrm{C}}$ and $V_{\mathrm{H}}$, are given by

$$
\begin{aligned}
\alpha_{\perp} & =\frac{a}{2 \eta} u_{\perp}\left(u_{\| \mathrm{C}} \phi_{\mathrm{C}}\left(u_{\perp} a / 2 \eta\right)+\frac{1}{2} u_{\| \mathrm{H}} \phi_{\mathrm{H}}\left(u_{\perp} a / 2 \eta\right)\right) \\
& =\frac{\eta}{a} R m_{\perp}\left(R m_{\| \mathrm{C}} \phi_{\mathrm{C}}\left(R m_{\perp}\right)+\frac{1}{2} R m_{\| \mathrm{H}} \phi_{\mathrm{H}}\left(R m_{\perp}\right)\right) \\
& =\frac{V_{\mathrm{H}}}{a^{2} h \eta}\left(V_{\mathrm{C}} \phi_{\mathrm{C}}\left(V_{\mathrm{H}} / h \eta\right)+\frac{1}{2} V_{\mathrm{H}} \phi_{\mathrm{H}}\left(V_{\mathrm{H}} / h \eta\right)\right) .
\end{aligned}
$$

The functions $\phi_{\mathrm{C}}$ and $\phi_{\mathrm{H}}$, which have to satisfy $\phi_{\mathrm{C}}(0)=\phi_{\mathrm{H}}(0)=1$, may depend, apart from the arguments, also on the profile of $\omega$.

In order to determine $\alpha_{\perp}$ and so $\phi_{\mathrm{C}}$ and $\phi_{\mathrm{H}}$ the Eqs. (13) have been solved numerically in the region $-a \leq x, y \leq a$ using proper periodic boundary conditions (Rädler and Brandenburg, 2002). For the sake of simplicity both $u$ and $\omega$ were taken as constants, that is, rigid-body motions of the fluid, or piston profiles, were assumed in each of the channels. Results for $\phi_{\mathrm{C}}\left(R m_{\perp}\right)$ and $\phi_{\mathrm{H}}\left(R m_{\perp}\right)$ obtained in this way are 


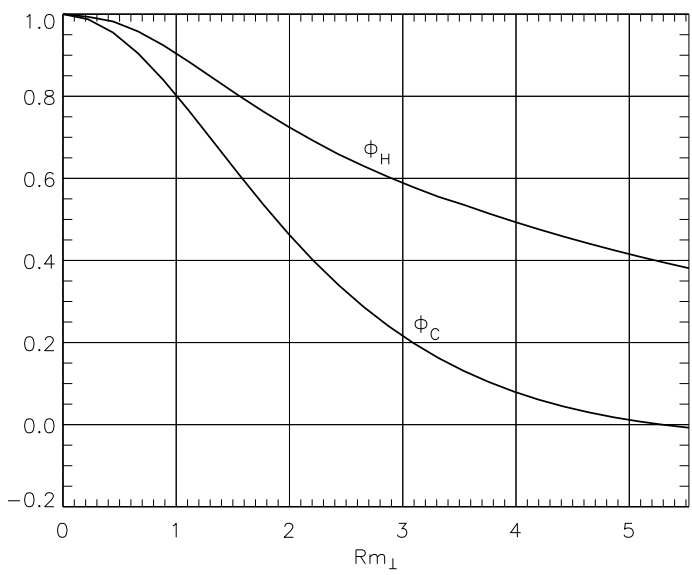

Fig. 9. The functions $\phi_{\mathrm{C}}\left(R m_{\perp}\right)$ and $\phi_{\mathrm{H}}\left(R m_{\perp}\right)$ for rigid-body motion of the fluid in each of the channels with $\varrho_{1}=a / 4, \varrho_{2}=a / 2$ and $h=0.905 a$

shown in Fig. 9. The dependence of $a^{2} \alpha_{\perp}$ on $V_{\mathrm{C}}$ and $V_{\mathrm{H}}$ for $\varrho_{1}=a / 4, \varrho_{2}=a / 2$ and $h=0.905 a$ (chosen in agreement with Eq. 32) is represented in Fig. 10. ${ }^{1}$ Again the comment given with Fig. 7 applies according to which there is a value $V_{\mathrm{C}}^{*}\left(\right.$ here $\left.V_{\mathrm{C}}^{*}<4\right)$ so that $\alpha_{\perp}\left(V_{\mathrm{C}}, V_{\mathrm{H}}\right)$ for any fixed $V_{\mathrm{C}}<V_{\mathrm{C}}^{*}$ grows with $V_{\mathrm{H}}$, for $V_{\mathrm{C}}>V_{\mathrm{C}}^{*}$, however, grows for small $V_{\mathrm{H}}$ only, then reaches a maximum and decays again for larger $V_{\mathrm{H}}$. We also refer to Fig. 12 which shows isolines of $C$, which because of $C=\alpha_{\perp} R / \eta$ can easily be interpreted as isolines of $\alpha_{\perp}$.

\section{The self-excitation condition of the experimental device in comparison with experimental results}

\subsection{Self-excitation condition}

In the following we will apply the results obtained so far to the experimental device and compare them with experimental findings. For this purpose we choose for the radius $R$ and the height $H$ of the dynamo module the values

$R=0.85 \mathrm{~m}, \quad H=0.71 \mathrm{~m}$.

More precisely, as indicated in Fig. 1 these values correspond to the "homogeneous part" of the dynamo module, which does not include the regions with connections between the spin generators, etc. We further adopt for the edge length $a$ of a spin generator, the radius $\varrho_{1}$ of the inner channel, the outer radius $\varrho_{2}$ and the pitch $h$ of the helical channel

$a=0.21 \mathrm{~m}, \varrho_{1}=0.25 a, \varrho_{2}=0.5 a, h=0.19 \mathrm{~m}$.

\footnotetext{
${ }^{1}$ The results presented in some of our earlier papers (Rädler et al., 1997a,b, 1998b) were obtained with an analytic solution of Eqs. (13) for a single spin generator ignoring the influence of the neighbouring ones, what is not completely correct beyond the second-order approximation. The numerical investigations mentioned confirm the essential features of the results but show that corrections of numerical data are necessary if $R m_{\perp}$ is no longer small compared to unity. These corrections are considered here.
}

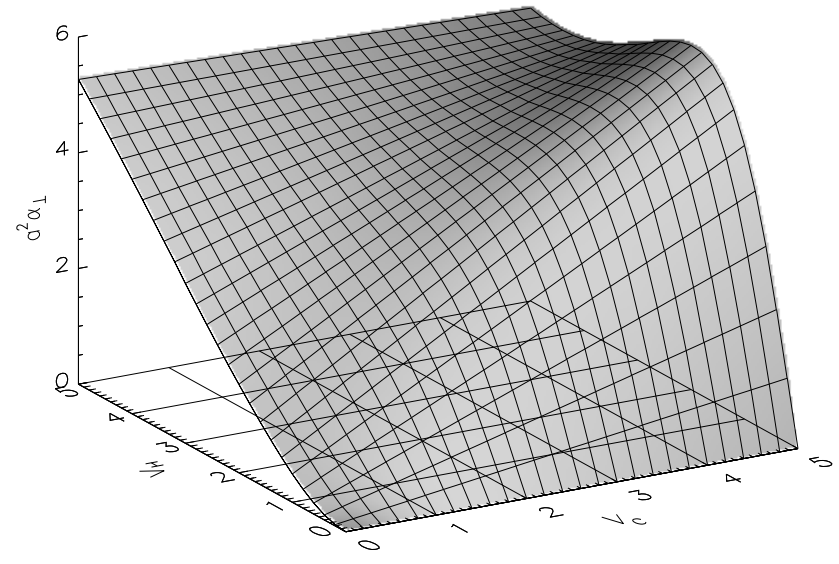

Fig. 10. The dependence of $a^{2} \alpha_{\perp}$ on $V_{\mathrm{C}}$ and $V_{\mathrm{H}}$, all three quantities measured in units of $a \eta$, with $\varrho_{1}=a / 4, \varrho_{2}=a / 2$ and $h=0.905 a$

Finally we put for the magnetic diffusivity of liquid sodium

$\eta=0.1 \mathrm{~m}^{2} / \mathrm{s}$.

We return first to the dimensionless measure $C$ of the $\alpha$ effect introduced with Eq. (12) and express it by the volumetric flow rates $V_{\mathrm{C}}$ and $V_{\mathrm{H}}$. With the result (21), which was obtained for the Roberts flow, we find the dependence of $C$ on $V_{\mathrm{C}}$ and $V_{\mathrm{H}}$ depicted in Fig. 11. In the same way the result (30) for the spin generator flow leads to the dependence shown in Fig. 12. For the Roberts flow we can show that, when admitting arbitrary $V_{\mathrm{C}}$ and $V_{\mathrm{H}}$, each isoline of $C$ cuts the $V_{\mathrm{H}}$-axis and continues until infinite $V_{\mathrm{C}}$. Presumably the same applies to the spin generator flow. The non-uniqueness of $V_{\mathrm{H}}$ as a function of $V_{\mathrm{C}}$ is, of course, again a consequence of the magnetic flux expulsion from the inner parts of the spin-generators. Remarkably enough, in the regions of $V_{\mathrm{C}}$ and $V_{\mathrm{H}}$ which are of interest for the experiment, that is $0<V_{\mathrm{C}}, V_{\mathrm{H}}<200 \mathrm{~m}^{3} / \mathrm{h}$, the isolines of $C$ essentially coincide for both kinds of flow patterns. Considering the spin generator flow as more realistic than the Roberts flow we will refer to the isolines of $C$ shown in Fig. 12 in what follows.

We recall that in our approach the self-excitation condition for the dynamo reads $C \geq C^{*}$ and that values of $C^{*}$ obtained under various assumptions are listed in Table 1. For any given value of $C^{*}$ we have a "neutral line" $C=C^{*}$ in the $V_{\mathrm{C}} V_{\mathrm{H}}$-plane separating the region in which the dynamo can work from the one where it can not. As can be seen from Figs. 11 and 12 dynamo action should be possible for arbitrarily small $V_{\mathrm{H}}$ if only $V_{\mathrm{C}}$ exceeds a sufficiently large value depending on $V_{\mathrm{H}}$. Likewise a dynamo should work with $V_{\mathrm{C}}=0$ and a sufficiently large $V_{\mathrm{H}}$.

\subsection{Experimental results}

Using data measured in the experiment (Müller and Stieglitz, private communication) the real neutral line in the $V_{\mathrm{C}} V_{\mathrm{H}^{-}}$ plane separating dynamo and non-dynamo regions has been determined. Figure 13 shows a detail of Fig. 12 with this 


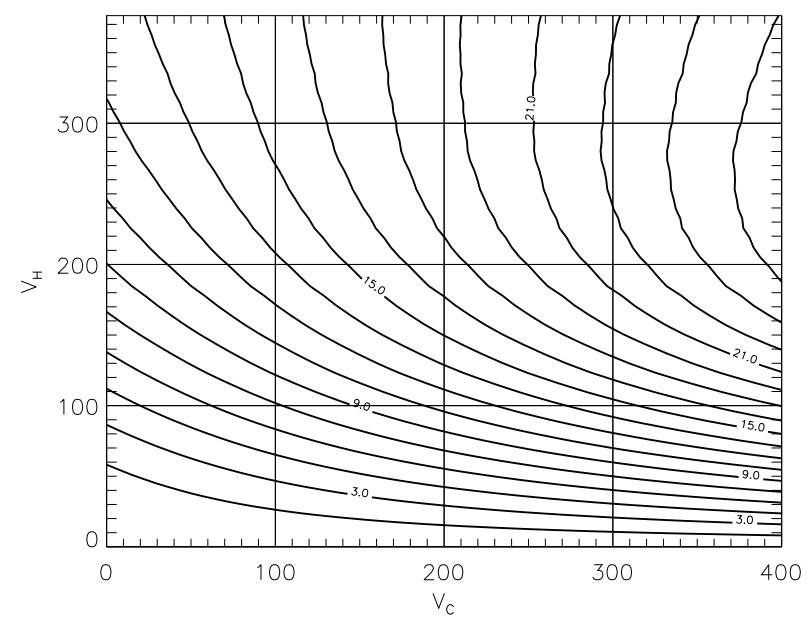

Fig. 11. Isolines of $C$, obtained with the result (21) for the Roberts

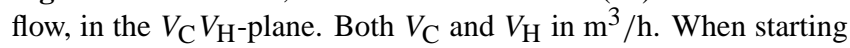
from the numerical values related to the units used in Fig. 7 those related to $\mathrm{m}^{3} / \mathrm{h}$ follow by multiplication by a factor 75.6 .

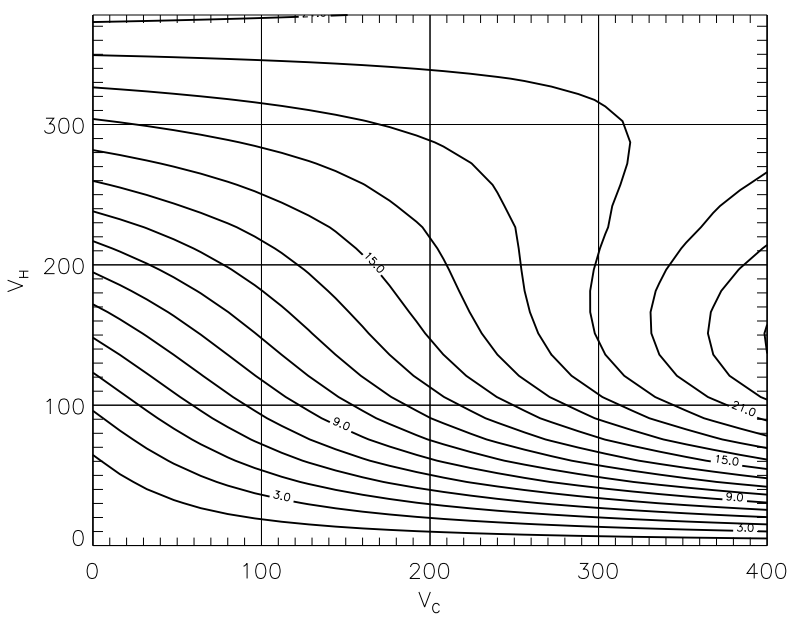

Fig. 12. Isolines of $C$, obtained with the result (30) for the spin generator flow, in the $V_{\mathrm{C}} V_{\mathrm{H}}$-plane. Both $V_{\mathrm{C}}$ and $V_{\mathrm{H}}$ in $\mathrm{m}^{3} / \mathrm{h}$. When starting from the numerical values related to the units used in Fig. 10 those related to $\mathrm{m}^{3} / \mathrm{h}$ follow by multiplication by a factor 75.6 .

line added. It corresponds to values of $C^{*}$ in the interval $8.4 \cdots 9.3$.

Magnetic field measurements have been carried out at several points along the axis of the dynamo module. It turned out that the field there consists mainly of $x$ - and $y$-components. Compared to them no noticeable $z$-component was observed. This applies likewise to the components of $\overline{\boldsymbol{B}}$ (see Appendix C) and indicates that, as expected, the generated fields are of the symmetry type $m=1$. It should, however, be noted that the variation of the $x$ - and $y$-components of $\bar{B}$ derived from the observed field along the axis of the dynamo module (see again Appendix C) are not in satisfactory agreement with calculated field structures as shown in Fig. 5.

The fact that the values of $C^{*}$ derived from the measure-

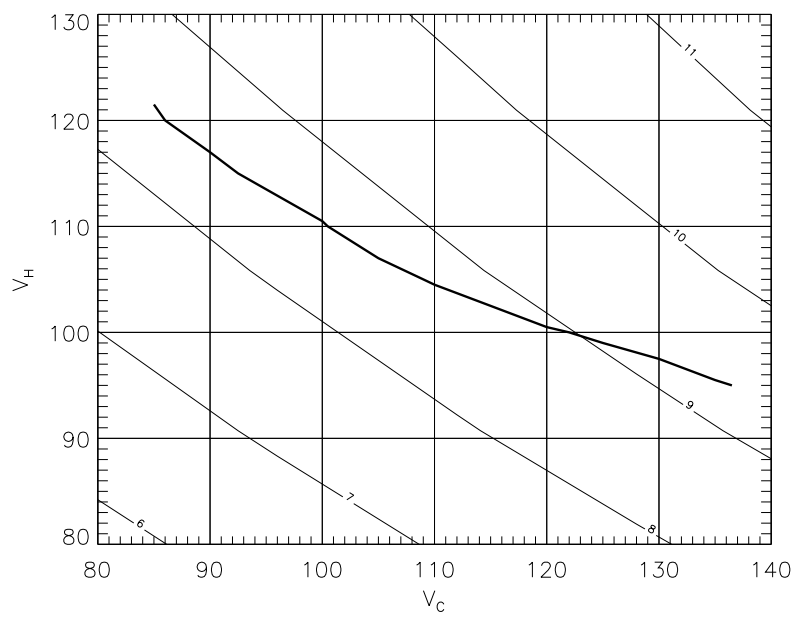

Fig. 13. A detail of Fig. 12 with the experimentally determined neutral line (the thick line) separating regions with and without dynamo action

ments are somewhat higher than those given for $m=1$ in Table 1 is well understandable. As we will explain in more detail below (Sect. 7) improvements to the simple models for which Table 1 applies lead to higher values of $C^{*}$. Such improvements consider in particular the variability of the coefficient $\alpha_{\perp}$ and the occurrence of effects described by $\alpha_{\|}$and $\gamma$ near the boundaries of the dynamo-active body as well as the effects described by $\beta_{\perp}, \beta_{\|}$and $\beta_{3}$ inside this body.

\section{On the back-reaction of the magnetic field on the fluid flow and the saturation of the magnetic field}

\subsection{A simple dynamo model involving the back-reaction of} the magnetic field

So far we considered kinematic dynamo models only, that is, we ignored the influence of the Lorentz forces on the fluid flows. The Lorentz forces are of second order in the magnetic field, and their influence on the fluid flow grows with the magnetic field and limits so its magnitude. In order to study this process in detail in addition to the induction equation (Eq. 1) the hydrodynamic equations involving the Lorentz forces have to be taken into account.

Instead of investigating the very complex problem which occurs in this way we deal here only with a simple model of the dynamo in the nonlinear regime (see also Rädler et al., 1998a). It considers no other consequence of the backreaction of the magnetic field on the fluid motion than the magnetic contribution to the pressure drops in the channels of the spin generators. Influences of the magnetic field on the flow profiles in the channels (as discussed in Rädler et al., 2000a) or the generation of motions in the fluid outside the channels are ignored.

We start again from Eqs. (10) for $\overline{\boldsymbol{B}}$, with $\alpha_{\perp}$ considered as a function of the flow rates $V_{\mathrm{C}}$ and $V_{\mathrm{H}}$, and add two equations for these quantities. The latter equations are to be understood 
as consequences of the Navier-Stokes equation, relating the flow rates to the pressures built up by the pumps and the pressure losses due to the hydraulic resistance and the magnetic field. The full set of these equations reads

$$
\begin{aligned}
& \partial_{t} \overline{\boldsymbol{B}}=\eta \nabla^{2} \overline{\boldsymbol{B}}+\alpha_{\perp}\left(V_{\mathrm{C}}, V_{\mathrm{H}}\right) \nabla \times(\overline{\boldsymbol{B}}-(\boldsymbol{e} \cdot \overline{\boldsymbol{B}}) \boldsymbol{e}), \\
& \nabla \cdot \overline{\boldsymbol{B}}=0, \\
& \mathrm{~d}_{t} V_{\mathrm{C}}=\kappa_{\mathrm{C}}\left(P_{\mathrm{C}}\left(V_{\mathrm{C}}\right)-R_{\mathrm{C}}\left(V_{\mathrm{C}}\right)-L_{\mathrm{C}}\left(\tilde{B}, V_{\mathrm{C}}, V_{\mathrm{H}}\right)\right), \\
& \mathrm{d}_{t} V_{\mathrm{H}}=\kappa_{\mathrm{H}}\left(P_{\mathrm{H}}\left(V_{\mathrm{H}}\right)-R_{\mathrm{H}}\left(V_{\mathrm{H}}\right)-L_{\mathrm{H}}\left(\tilde{B}, V_{\mathrm{C}}, V_{\mathrm{H}}\right)\right),
\end{aligned}
$$

where, of course, the first line must be completed using proper boundary conditions. Here $\kappa_{\mathrm{C}}$ and $\kappa_{\mathrm{H}}$ are factors of the structure $s / \rho_{\mathrm{m}} l$ where $s$ is the cross-section of the considered type of channels, $\rho_{\mathrm{m}}$ the mass density of the fluid and $l$ the total length of the considered circuit. $P_{\mathrm{C}}$ and $P_{\mathrm{H}}$ are the pressures generated by the pumps in these circuits, $R_{\mathrm{C}}$ and $R_{\mathrm{H}}$ the pressure drops due to the hydraulic resistances, $L_{\mathrm{C}}$ and $L_{\mathrm{H}}$ the pressure drops due to Lorentz forces, and $\tilde{B}$ is a quantity depending on the magnitude of the relevant components of the magnetic field, which will be specified later. We point out that according to our above assumption $\alpha_{\perp}$ depends only via $V_{\mathrm{C}}$ and $V_{\mathrm{H}}$ on the magnetic field, that is, a possible dependence via the flow profiles is not taken into account.

It should be noted that there is one circuit in the experimental device which contains the central channels of all 52 spin generators but there are two circuits for the helical channels, each feeding 26 of them. Here these two circuits are assumed to be equal to each other, that is, described by one flow rate, $V_{\mathrm{H}}$, only.

We specify Eq. (34) by further assumptions concerning $P_{\mathrm{C}}, P_{\mathrm{H}}, R_{\mathrm{C}}, R_{\mathrm{H}}, L_{\mathrm{C}}$ and $L_{\mathrm{H}}$. For the pressures generated by the pumps we put

$$
P_{\mathrm{C}}=k_{\mathrm{C}} P_{\mathrm{C}}^{o}\left(1-c_{\mathrm{PC}} V_{\mathrm{C}}\right), P_{\mathrm{H}}=k_{\mathrm{H}} P_{\mathrm{H}}^{o}\left(1-c_{\mathrm{PH}} V_{\mathrm{H}}\right),
$$

where the factors $k_{\mathrm{C}}$ and $k_{\mathrm{H}}$ describe with which fractions of the maximum pressure the pumps work, $0<k_{\mathrm{C}}, k_{\mathrm{H}} \leq 1$. Further, $P_{\mathrm{C}}^{o}$ and $P_{\mathrm{H}}^{o}$ are the maximum pressures, and $c_{\mathrm{PC}}$ and $c_{\mathrm{PH}}$ are constants considering the pressure drops inside the pumps under load (see Stieglitz and Müller, 1996). For the pressure losses due to the hydraulic resistance we assume

$$
R_{\mathrm{C}}=R_{\mathrm{C}}^{o}\left(1+c_{\mathrm{RC}}\left(1+\frac{c_{\mathrm{RC}}^{\prime}}{V_{\mathrm{C}}}\right)^{1 / 4}\right) V_{\mathrm{C}}^{2}, R_{\mathrm{H}}=R_{\mathrm{H}}^{o} V_{\mathrm{H}}^{2},
$$

where $R_{\mathrm{C}}^{o}, R_{\mathrm{H}}^{o}, c_{\mathrm{RC}}$ and $c_{\mathrm{RC}}^{\prime}$ are constants (see again Stieglitz and Müller, 1996). The main contributions to the resistances are due to the bends of the tubes.

Corresponding relations for $L_{\mathrm{C}}$ and $L_{\mathrm{H}}$ will be given below.

\subsection{Estimates of the Lorentz forces}

For an estimate of the Lorentz forces we assume that $\overline{\boldsymbol{B}}$ is a homogeneous field. Then the force exerted on a unit volume of the fluid, $f$, is given by

$\boldsymbol{f}=\frac{1}{\mu_{0}}\left(\nabla \times \boldsymbol{B}^{\prime}\right) \times\left(\overline{\boldsymbol{B}}+\boldsymbol{B}^{\prime}\right)$, where $\mu_{0}$ is the magnetic permeability of free space.

We restrict ourselves first to the second-order approximation as explained in the context of Eq. (13) and replace in the same sense $\overline{\boldsymbol{B}}+\boldsymbol{B}^{\prime}$ in Eq. (37) simply by $\overline{\boldsymbol{B}}$. For the calculation of the averages of $\boldsymbol{f}$ which are of interest below it is then again justified to consider a single spin generator only, that is, to ignore any motion in the neighbouring ones. As in Sect. 4.3 we consider the spin-generator defined by $0 \leq x, y \leq a$ and use again the co-ordinate system $\varrho, \varphi$, $z$ introduced there. We can easily find a steady solution $\boldsymbol{B}^{\prime}$ of Eq. (13) (see Appendix A) and calculate $\boldsymbol{f}$ according to Eq. (37). Averaging its $\varphi$ and $z$-components over $\varphi$ and denoting these averages by $\hat{f}_{\varphi}$ and $\hat{f}_{z}$ we have

$\hat{f}_{\varphi}=-\frac{1}{2} \sigma u_{\varphi} B_{\perp}^{2}, \quad \hat{f}_{z}=-\frac{1}{2} \sigma u_{z} B_{\perp}^{2}$,

where $\sigma$ is the electric conductivity of the fluid, $\sigma=1 / \mu_{0} \eta$, and $B_{\perp}$ the mean magnetic flux density in the $x y$-plane. Of course, $\hat{f}_{\varphi}$ and $\hat{f}_{z}$ depend on $\varrho$ if $u_{\varphi}$ and $u_{z}$ do so.

Consider first a central channel. The magnetic pressure drop per unit length $\left(\mathrm{d} p_{\mathrm{m}} / \mathrm{d} l\right)_{\mathrm{C}}$ of this channel is, apart from the sign, just the average of $\hat{f}_{z}$ over its cross-section, that is

$\left(\frac{\mathrm{d} p_{\mathrm{m}}}{\mathrm{d} l}\right)_{\mathrm{C}}=\frac{1}{2} \sigma\left\langle u_{z}\right\rangle B_{\perp}^{2}$,

where $\left\langle u_{z}\right\rangle$ means the average of $u_{z}$ over the volume or, what is the same, over the cross-section of the channel. Denoting this cross-section by $s_{\mathrm{C}}$, where $s_{\mathrm{C}}=\pi \varrho_{1}^{2}$, and using $\left\langle u_{z}\right\rangle s_{\mathrm{C}}=a^{2} u_{\| \mathrm{C}}$ we find further

$$
\left(\frac{\mathrm{d} p_{\mathrm{m}}}{\mathrm{d} l}\right)_{\mathrm{C}}=\frac{\sigma a^{2} B_{\perp}^{2}}{2 s_{\mathrm{C}}} u_{\| \mathrm{C}}=\frac{a B_{\perp}^{2}}{2 \mu_{0} s_{\mathrm{C}}} R m_{\| \mathrm{C}}=\frac{\sigma B_{\perp}^{2}}{2 s_{\mathrm{C}}} V_{\mathrm{C}} .
$$

Consider next a helical channel. For the pressure drop per unit length $\left(\mathrm{d} p_{\mathrm{m}} / \mathrm{d} l\right)_{\mathrm{H}}$ we can derive a relation analogous to Eq. (39) with $\left\langle u_{z}\right\rangle$ replaced by $\cos \delta\left\langle u_{\varphi}\right\rangle+\sin \delta\left\langle u_{z}\right\rangle$. Here $\delta$ means the angle between some central stream line at a radius $\varrho=\bar{\varrho}$ and a circle with $\varrho=\bar{\varrho}$ and $z=$ const, that is, $\tan \delta=h / 2 \pi \bar{Q}$, and $\left\langle u_{\varphi}\right\rangle$ and $\left\langle u_{z}\right\rangle$ are now averages over the volume of the channel or, what is the same, over its section with a plane $z=$ const. We define $\varrho$ by $\int_{\varrho_{1}}^{\varrho_{2}} u_{\varphi}(\varrho) \varrho d \varrho=$ $\bar{\varrho} \int_{\varrho_{1}}^{\varrho_{2}} u_{\varphi}(\varrho) d \varrho$ and put $\bar{\varrho}=\xi^{\prime}\left(\varrho_{1}+\varrho_{2}\right) / 2$ where $\xi^{\prime}$ is a factor close to unity. Further we introduce the cross-section $s_{\mathrm{H}}$ as the area of a plane fitting into the channel and being perpendicular to the central stream line mentioned, that is $s_{\mathrm{H}}=\left(\varrho_{2}-\varrho_{1}\right) h \cos \delta$. So we arrive at

$$
\begin{aligned}
& \left(\frac{\mathrm{d} p_{\mathrm{m}}}{\mathrm{d} l}\right)_{\mathrm{H}}=\frac{\sigma a h \xi^{\prime} B_{\perp}^{2}}{4 s_{\mathrm{H}}} u_{\perp} \\
& =\frac{h \xi^{\prime} B_{\perp}^{2}}{2 \mu_{0} s_{\mathrm{H}}} R m_{\perp}=\frac{\sigma \xi^{\prime} B_{\perp}^{2}}{2 s_{\mathrm{H}}} V_{\mathrm{H}} .
\end{aligned}
$$

Let us now leave the second-order approximation in Eq. (13) and the analogous one in Eq. (37). Using analytical solutions of Eq. (13) for an isolated spin generator, that is, ignoring as before the influences of the neighbouring 


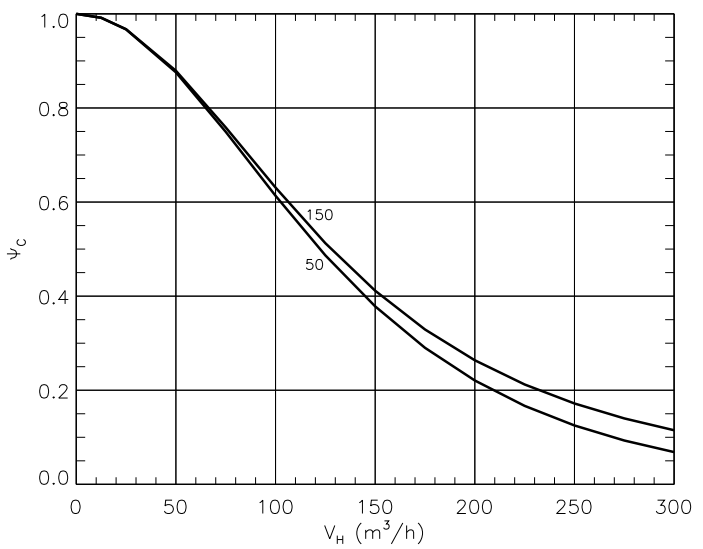

Fig. 14. The function $\psi_{\mathrm{C}}\left(V_{\mathrm{C}}, V_{\mathrm{H}}\right)$ for two special values of $V_{\mathrm{C}}$ given by the labels of the curves, with $\varrho_{1}=a / 4$ and $\varrho_{2}=a / 2$. Both $V_{\mathrm{C}}$ and $V_{\mathrm{H}}$ in $\mathrm{m}^{3} / \mathrm{h}$. The function $\psi_{\mathrm{C}}$ varies monotonically with $V_{C}$.

ones, and assuming rigid-body motions, that is piston profiles, $\left(\mathrm{d} p_{\mathrm{m}} / \mathrm{d} l\right)_{\mathrm{C}}$ and $\left(\mathrm{d} p_{\mathrm{m}} / \mathrm{d} l\right)_{\mathrm{H}}$ for both channels have been determined for arbitrary $V_{\mathrm{C}}$ and $V_{\mathrm{H}}$. We present the result in the form

$$
\begin{aligned}
& \left(\frac{\mathrm{d} p_{\mathrm{m}}}{\mathrm{d} l}\right)_{\mathrm{C}}=\frac{\sigma B_{\perp}^{2}}{2 s_{\mathrm{C}}} V_{\mathrm{C}} \psi_{\mathrm{C}}\left(V_{\mathrm{C}}, V_{\mathrm{H}}\right) \\
& \left(\frac{\mathrm{d} p_{\mathrm{m}}}{\mathrm{d} l}\right)_{\mathrm{H}}=\frac{\sigma \xi^{\prime} B_{\perp}^{2}}{2 s_{\mathrm{H}}} V_{\mathrm{H}} \psi_{\mathrm{H}}\left(V_{\mathrm{C}}, V_{\mathrm{H}}\right),
\end{aligned}
$$

with two functions $\psi_{\mathrm{C}}$ and $\psi_{\mathrm{H}}$ satisfying $\psi_{\mathrm{C}}\left(V_{\mathrm{C}}, 0\right)=$ $\psi_{\mathrm{H}}\left(V_{\mathrm{C}}, 0\right)=1$. These functions with $\varrho_{1}=a / 4$ and $\varrho_{2}=a / 2$ are shown in Figs. 14 and 15.

We complete now the Eqs. (34) to (36) by

$$
\begin{array}{ll}
L_{\mathrm{C}}=B_{\perp}^{2} \tilde{L}_{\mathrm{C}}, & \tilde{L}_{\mathrm{C}}=c_{\mathrm{LC}} V_{\mathrm{C}} \psi_{\mathrm{C}}\left(V_{\mathrm{C}}, V_{\mathrm{H}}\right) \\
L_{\mathrm{H}}=B_{\perp}^{2} \tilde{L}_{\mathrm{H}}, & \tilde{L}_{\mathrm{H}}=c_{\mathrm{LH}} V_{\mathrm{H}} \psi_{\mathrm{H}}\left(V_{\mathrm{C}}, V_{\mathrm{H}}\right)
\end{array}
$$

where $c_{\mathrm{LC}}$ and $c_{\mathrm{LH}}$ are constants of the structure $\sigma l / 2 s$, with $\sigma, l$ and $s$ being the electric conductivity of the fluid, the total length of all channels in the considered circuit and $s$ their cross-section.

\subsection{Saturated dynamo states}

We consider now our dynamo model defined by the Eq. (34) together with Eqs. (35), (36) and (43) for a state in which $\overline{\boldsymbol{B}}, V_{\mathrm{C}}$ and $V_{\mathrm{H}}$ neither grow nor decay. We already know from kinematic dynamo models that $\bar{B}$ for fixed $V_{\mathrm{C}}$ and $V_{\mathrm{H}}$ shows a non-oscillatory behaviour, and we could not find any example of a different behaviour of $\overline{\boldsymbol{B}}, V_{\mathrm{C}}$ and $V_{\mathrm{H}}$ in the case considered here. Therefore we restrict our attention here to the steady case.

Steady solutions of the equations for $\bar{B}$ in Eq. (34) require that $C\left(V_{\mathrm{C}}, V_{\mathrm{H}}\right)$ takes its marginal value $C^{*}$. Hence the consequences of the Eqs. (34) with (35), (36) and (43) for the steady case read

$C\left(V_{\mathrm{C}}, V_{\mathrm{H}}\right)=C^{*}$

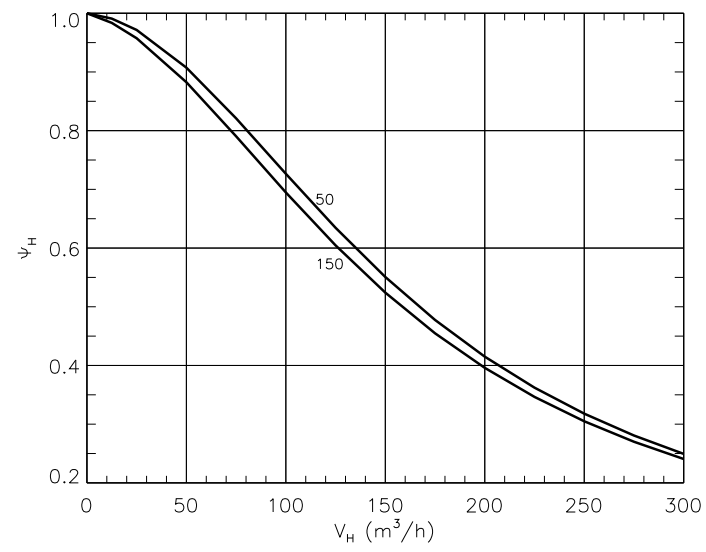

Fig. 15. The function $\psi_{\mathrm{H}}\left(V_{\mathrm{C}}, V_{\mathrm{H}}\right)$ for two special values of $V_{\mathrm{C}}$ given by the labels of the curves, with $\varrho_{1}=a / 4$ and $\varrho_{2}=a / 2$. Both $V_{\mathrm{C}}$ and $V_{\mathrm{H}}$ in $\mathrm{m}^{3} / \mathrm{h}$. The function $\psi_{\mathrm{H}}$ varies monotonically with $V_{C}$.

$P_{\mathrm{C}}\left(V_{\mathrm{C}}\right)-R_{\mathrm{C}}\left(V_{\mathrm{C}}\right)-B_{\perp}^{2} \tilde{L}_{\mathrm{C}}\left(V_{\mathrm{C}}, V_{\mathrm{H}}\right)=0$
$P_{\mathrm{H}}\left(V_{\mathrm{H}}\right)-R_{\mathrm{H}}\left(V_{\mathrm{H}}\right)-B_{\perp}^{2} \tilde{L}_{\mathrm{H}}\left(V_{\mathrm{C}}, V_{\mathrm{H}}\right)=0$.

Eliminating $B_{\perp}^{2}$ from the last two lines of Eq. (44) we find

$$
\begin{aligned}
& \left(P_{\mathrm{C}}\left(V_{\mathrm{C}}\right)-R_{\mathrm{C}}\left(V_{\mathrm{C}}\right)\right) \tilde{L}_{\mathrm{H}}\left(V_{\mathrm{C}}, V_{H}\right) \\
& \quad-\left(P_{\mathrm{H}}\left(V_{\mathrm{H}}\right)-R_{\mathrm{H}}\left(V_{\mathrm{H}}\right)\right) \tilde{L}_{\mathrm{C}}\left(V_{\mathrm{C}}, V_{\mathrm{H}}\right)=0 .
\end{aligned}
$$

If all other relevant parameters are given the first line of Eq. (44) together with Eq. (45) allows us to determine a pair, or possibly several pairs, of values $V_{\mathrm{C}}$ and $V_{\mathrm{H}}$ without considering $B_{\perp}^{2}$. With the help of the second or the third line of Eq. (44) we can afterwards find the corresponding value of $B_{\perp}^{2}$. We must, however, discard all pairs of $V_{\mathrm{C}}$ and $V_{\mathrm{H}}$ for which $B_{\perp}^{2}$ takes negative values.

On the basis of Eq. (44), completed by Eqs. (35), (36) and (43), we may calculate the quantities $V_{\mathrm{C}}, V_{\mathrm{H}}$ and $B_{\perp}$ if, for example, $C^{*}, k_{\mathrm{C}}$ and $k_{\mathrm{H}}$ are given. For this purpose we need the numerical values of $P_{\mathrm{C}}^{o}, P_{\mathrm{H}}^{o}, c_{\mathrm{PC}}, c_{\mathrm{PH}}, R_{\mathrm{C}}^{o}, R_{\mathrm{H}}^{o}, c_{\mathrm{RC}}$, $c_{\mathrm{RH}}^{\prime}, c_{\mathrm{LC}}$ and $c_{\mathrm{LH}}$. Without going into details we note that the parameters of the device (see Stieglitz and Müller, 1996) lead to

$P_{\mathrm{C}}^{o}=P_{\mathrm{H}}^{o}=710 \mathrm{kPa}, c_{\mathrm{PC}}=c_{\mathrm{PH}}=10.1\left(\mathrm{~m}^{3} / \mathrm{s}\right)^{-1}$

$R_{\mathrm{C}}^{o}=1.31 \cdot 10^{8} \mathrm{~Pa}\left(\mathrm{~m}^{3} / \mathrm{s}\right)^{-2}, R_{\mathrm{H}}^{o}=1.99 \cdot 10^{8} \mathrm{~Pa}\left(\mathrm{~m}^{3} / \mathrm{s}\right)^{-2}$

$c_{\mathrm{RC}}=3.54 \cdot 10^{-1}, c_{\mathrm{RC}}^{\prime}=3.54 \cdot 10^{-2} \mathrm{~m}^{3} / \mathrm{s}$

$c_{\mathrm{LC}}=1.88 \cdot 10^{10} \mathrm{~kg}\left(\mathrm{~m}^{4} \mathrm{sT}^{2}\right)^{-1}$,

$c_{\mathrm{LH}}=2.31 \cdot 10^{10} \mathrm{~kg}\left(\mathrm{~m}^{4} \mathrm{sT}^{2}\right)^{-1}$

(see also Rädler et al., 2000a,b).

In Table 2 flow rates $V_{\mathrm{C}}$ and $V_{\mathrm{H}}$ and the quantity $B_{\perp}$ characterizing the magnitude of the generated magnetic field in steady states of the dynamo are listed for various values of $C^{*}, k_{\mathrm{C}}$ and $k_{\mathrm{H}}$. In addition, the total power $N$ is given which is needed to maintain these states as well as the relative fraction $f_{\text {ohm }}$ fed into the magnetic field and converted into heat by ohmic dissipation. Since some of the numerical values 
Table 2. The flow rates $V_{\mathrm{C}}$ and $V_{\mathrm{H}}$ and the measure $B_{\perp}$ of the magnitude of the magnetic field for steady states of the dynamo, further the total power $N$ needed to maintain these steady states and its relative fraction $f_{\text {ohm }}$ corresponding to ohmic dissipation

\begin{tabular}{cccccccc}
\hline$C^{*}$ & $k_{\mathrm{C}}$ & $k_{\mathrm{H}}$ & $\begin{array}{c}V_{\mathrm{C}} \\
{\left[\mathrm{m}^{3} / \mathrm{h}\right]}\end{array}$ & $\begin{array}{c}V_{\mathrm{H}} \\
{\left[\mathrm{m}^{3} / \mathrm{h}\right]}\end{array}$ & $\begin{array}{c}B_{\perp} \\
{\left[10^{-4} T\right]}\end{array}$ & $\begin{array}{c}N \\
{[\mathrm{~kW}]}\end{array}$ & $f_{\text {ohm }}$ \\
\hline 8.0 & 1 & 1 & 106 & 92 & 303 & 42 & 0.73 \\
& 1 & 0.5 & 127 & 78 & 215 & 28 & 0.57 \\
& 0.5 & 1 & 84 & 108 & 256 & 36 & 0.63 \\
& 0.5 & 0.5 & 102 & 94 & 169 & 21 & 0.45 \\
\hline 8.5 & 1 & 1 & 110 & 96 & 291 & 43 & 0.70 \\
& 1 & 0.5 & 130 & 82 & 204 & 29 & 0.52 \\
& 0.5 & 1 & 89 & 113 & 243 & 37 & 0.59 \\
& 0.5 & 0.5 & 106 & 98 & 155 & 21 & 0.40 \\
\hline 9.0 & 1 & 1 & 114 & 100 & 281 & 44 & 0.67 \\
& 1 & 0.5 & 134 & 86 & 193 & 29 & 0.49 \\
& 0.5 & 1 & 93 & 118 & 230 & 38 & 0.54 \\
& 0.5 & 0.5 & 110 & 103 & 140 & 22 & 0.33 \\
\hline 9.5 & 1 & 1 & 118 & 104 & 270 & 45 & 0.64 \\
& 1 & 0.5 & 138 & 90 & 182 & 30 & 0.44 \\
& 0.5 & 1 & 97 & 123 & 216 & 39 & 0.49 \\
& 0.5 & 0.5 & 114 & 108 & 123 & 22 & 0.26
\end{tabular}

in Eq. (46) have noticeable uncertainties, the values of $B_{\perp}$, $N$ and $f_{\text {ohm }}$ must be considered as rough estimates. In this sense they are in good agreement with the experimental results.

\section{Steps toward a refined theory of the experiment}

\subsection{Boundary effects}

7.1.1 The plane bottom and top boundaries of the dynamo module

The calculations of the electromotive force $\mathcal{E}$ reported above ignored the fact that the fluid flow is restricted to the dynamo module and that near its bottom and top covers there are flows between the spin generators. In order to get an idea on the influence of this type of boundary effect on the excitation condition of the dynamo, a calculation of the coefficients $\alpha_{\perp}, \alpha_{\|}$and $\gamma$ occurring in Eq. (8) has been carried out in the second-order approximation no longer assuming a Roberts flow as given by Eq. (14) but the modified flow defined by

$\boldsymbol{u}=u_{\perp} \frac{a}{2} \boldsymbol{e} \times \nabla\left(f_{\perp} \chi\right)+u_{\|}\left(\frac{a}{2}\right)^{2} \nabla \times\left(\boldsymbol{e} \times \nabla\left(f_{\|} \chi\right)\right)$,

$\chi=\sin \left(\frac{\pi}{a} x\right) \sin \left(\frac{\pi}{a} y\right)$,

where $f_{\perp}$ and $f_{\|}$are functions of $z$ (see Rädler et al., 1996). With $f_{\perp}=f_{\|}=1$ we return to Eq. (14). We think, however, of functions $f_{\perp}$ and $f_{\|}$which are equal to unity in some inner part of the dynamo module only but decay with growing $|z|$

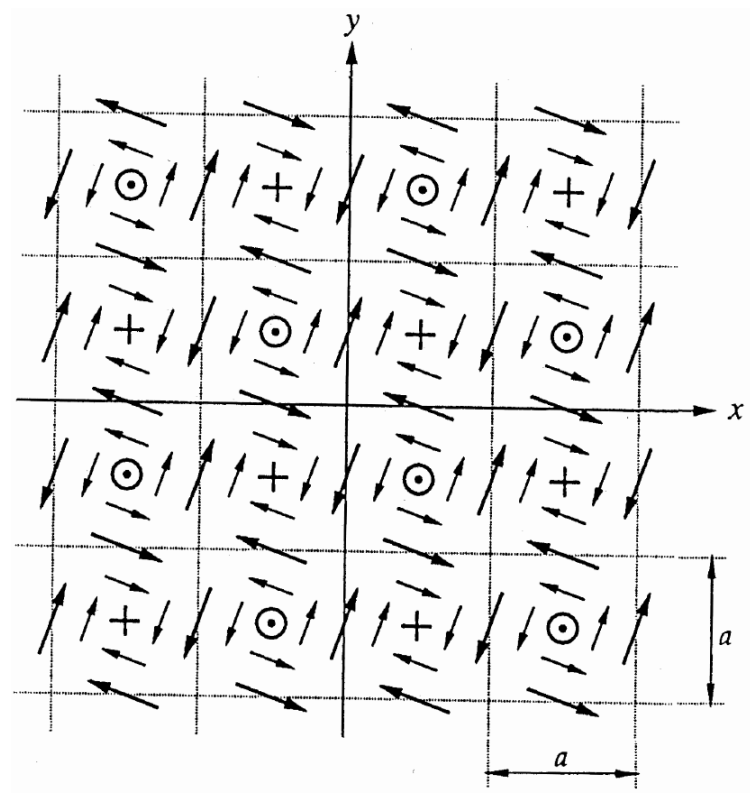

Fig. 16. A flow pattern with connecting flows between the spin generators

and vanish outside the module. A flow pattern in a region with varying $f_{\perp}$ and $f_{\|}$is shown in Fig. 16. With this flow $\alpha_{\perp}$ is no longer independent of $z$, and $\alpha_{\|}$and $\gamma$ are unequal to zero in and near to the regions with varying $f_{\perp}$ and $f_{\|}$. Calculations of these coefficients have been carried out for the two cases in which there is either free space beyond the covers of the dynamo module or a medium at rest with the same electric conductivity as the fluid (Rädler et al., 1996). We may represent the results in the form

$$
\begin{aligned}
& \alpha_{\perp}=\frac{\pi^{2}}{32} \frac{a}{\eta} u_{\perp} u_{\|} h_{\perp}(\zeta)=\frac{\pi^{2}}{16} \frac{\eta}{a} R m_{\perp} R m_{\|} h_{\perp}(\zeta) \\
& \alpha_{\|}=\frac{\pi^{2}}{32} \frac{a}{\eta} u_{\perp} u_{\|} h_{\|}(\zeta)=\frac{\pi^{2}}{16} \frac{\eta}{a} R m_{\perp} R m_{\|} h_{\|}(\zeta) \\
& \gamma=\frac{\pi^{2}}{16} \frac{a}{\eta} u_{\|}^{2} k(\zeta)=\frac{\pi^{2}}{16} \frac{\eta}{a} R m_{\|}^{2} k(\zeta),
\end{aligned}
$$

with the dimensionless functions $h_{\perp}, h_{\|}$and $k$ of $\zeta=2 z / H$. If $f_{\perp}$ and $f_{\|}$are symmetric in $z$ then $h_{\perp}$ and $h_{\|}$are again symmetric but $k$ is antisymmetric in $\zeta$.

Let us consider the simple example in which $f_{\perp}=1$ in $0 \leq|\zeta| \leq 1$, further $f_{\|}=1$ in $0 \leq|\zeta| \leq 1-\epsilon, f_{\|}=p_{5}(|\zeta|)$ in $1-\epsilon \leq|\zeta| \leq 1$, and $f_{\perp}=f_{\|}=0$ for $|\zeta| \geq 1$, where $\epsilon$ is a constant and $p_{5}$ a polynomial of the fifth degree such that $f_{\|}$and its first and second derivatives are continuous everywhere. The profiles of $h_{\perp}, h_{\|}$and $k$ for the case of free space beyond the covers of the dynamo module are shown in Fig. 17. Those for the case with a fluid at rest are very similar. Note that the sign of $k$ corresponds to a transport of magnetic flux out of the dynamo module.

The influence of the connecting flows in the sense discussed so far, that is, of the reduction of $\alpha_{\perp}$ and the occurrence of non-zero $\alpha_{\|}$and $\gamma$ in the boundary layers, on the 


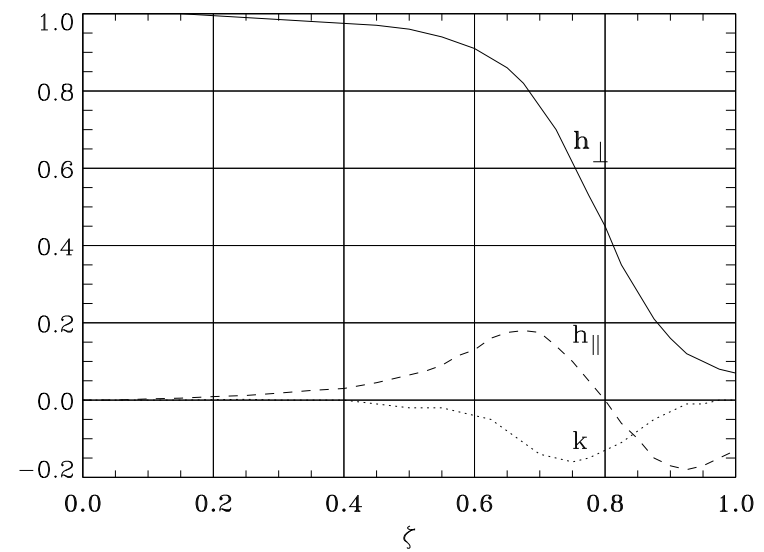

Fig. 17. The functions $h_{\perp}, h_{\|}$and $k$ for $f_{\perp}$ and $f_{\|}$as described in the text and $\epsilon=0.4$ in the case of free space beyond the covers of the dynamo module

self-excitation of the dynamo has been studied with a simple model in which the dynamo acts in an infinite slab surrounded by free space (Rädler et al., 1996). In this model the marginal value $C^{*}$, here related to a definition of $C$ analogous to Eq. (12) but with the thickness of the slab instead of $R$, depends on $\epsilon$ as introduced above and on $q=R m_{\|} / R m_{\perp}$, too. Compared to the case with constant $\alpha_{\perp}$ and vanishing $\alpha_{\|}$ and $\gamma$, the value of $C^{*}$ grows both with $\epsilon$ and $q$. For $\epsilon \leq 0.2$ and $q \leq 1$ the increase is less than $10 \%$. We may expect that in the experimental device the boundary effects discussed so far let $C^{*}$, compared to the idealized case, grow to a similar extent.

\subsubsection{The curved boundary of the dynamo module}

It is difficult to determine the mean velocity $\overline{\boldsymbol{u}}$ of the fluid or the mean electromotive force $\mathcal{E}$ for the curved boundary regions of the dynamo module. In any case $\overline{\boldsymbol{u}}$ must deviate from zero, and it must vary with the azimuth $\varphi$ with the pe$\operatorname{riod} \pi$. Likewise the coefficients of $\mathcal{E}$ as, e.g., $\alpha_{\perp}$ must show such a variation with $\varphi$. It is the neglect of these boundary effects which made that in the mean-field approach considered so far the dynamo module appeared to be an axisymmetric object and, as a consequence, there was no coupling between $\bar{B}$-fields differing in $m$. Of course, in a more detailed theory this axisymmetry of the dynamo module and its consequences must disappear. In the experiment indeed a clearly preferred direction for the generated fields occurs (Müller and Stieglitz, private communication).

\subsection{Mean-field conductivity, etc.}

So far we have not considered the contributions to the electromotive force $\mathcal{E}$ which are connected with derivatives of $\overline{\boldsymbol{B}}$. Dealing now with these contributions, we again restrict ourselves for the sake of simplicity to the case in which the flow pattern is independent of $z$, that is, to the $\beta_{\perp}, \beta_{\|}$and $\beta_{3}$ terms in Eq. (9). As already mentioned (Sect. 2) the first two can be interpreted in the sense that they contribute to a meanfield conductivity, and it is to be expected that they lead to an enhanced dissipation of the mean magnetic field. However, the last one does not need to act in this sense. A straightforward calculation with the Roberts flow defined by Eq. (14) using the second-order approximation yields

$$
\begin{gathered}
\beta_{\perp}=\frac{a^{2}}{64 \eta}\left(u_{\perp}^{2}+\frac{\pi^{2}}{4} u_{\|}^{2}\right)=\frac{\eta}{16}\left(R m_{\perp}^{2}+\frac{\pi^{2}}{16} R m_{\|}^{2}\right) \\
\beta_{\|}=\frac{a^{2}}{32 \eta} u_{\perp}^{2}=\frac{\eta}{8} R m_{\perp}^{2} \\
\beta_{3}=-\frac{a^{2}}{64 \eta}\left(u_{\perp}^{2}-\frac{\pi^{2}}{4} u_{\|}^{2}\right)=-\frac{\eta}{16}\left(R m_{\perp}^{2}-\frac{\pi^{2}}{16} R m_{\|}^{2}\right)
\end{gathered}
$$

(see Rädler et al., 1996). Note that $\beta_{\perp}$ and $\beta_{\|}$are positive definite, that is, must indeed lead to an enhanced dissipation of the mean magnetic field, whereas $\beta_{3}$ may take both signs so that it is difficult to predict its influence. Note also that we have $\beta_{3}=\beta_{\perp}-\beta_{\|}$.

The $\beta_{\perp}$ and $\beta_{\|}$-effects necessarily lead to higher values of $C^{*}$ for any given dynamo model. Estimates with a very simple model show that this tendency is maintained if in addition the $\beta_{3}$-effect is taken into account (Rädler et al., 1996). Again an increase of $C^{*}$ up to $10 \%$ is to be expected as a consequence of the effects discussed here. This statement is in agreement with results of another way of calculating the $\alpha_{\perp}$-effect and the $\beta_{\perp}, \beta_{\|}$and $\beta_{3}$-effects and their influences on $C^{*}$ (Rädler and Brandenburg, 2002).

\subsection{On the limits of the mean-field approach}

As usual in mean-field dynamo theory we have adopted the assumption that $\overline{\boldsymbol{B}}$ varies weakly in space and time so that all contributions to $\mathcal{E}$ with higher than first-order spatial derivatives and with any time derivatives of $\overline{\boldsymbol{B}}$ are negligible. We may consider the radius $R$ of the dynamo module as a characteristic length scale of $\bar{B}$ and the edge length $a$ of a spin generator as the averaging length scale. According to Eqs. (31) and (32) we have $a / R=0.25$. That is, the above assumption is not well satisfied and the statements derived from mean-field considerations should be checked in an independent way.

In this context investigations of subharmonic solutions of the original Roberts dynamo problem (Tilgner and Busse, 1995; Plunian and Rädler, 2002) are of interest. We rely here on the recent one of them (Plunian and Rädler, 2002), which is widely elaborated in view of the Karlsruhe dynamo, and adopt the definitions introduced above for the original $z$-independent Roberts flow. In particular such subharmonic solutions of the induction equation for $\boldsymbol{B}$ with $\boldsymbol{u}$ specified by Eq. (14) have been considered which possess no part independent of $x$ and $y$ and whose period lengths in $x$ - and $y$-direction are integer multiples of the length of a diagonal of a cell in the flow pattern, that is, $\sqrt{2} \mathrm{Na}$ with an integer $\mathrm{N}$. An arbitrary period length in $z$-direction was admitted, here denoted by $\sqrt{2} \kappa a$ with an arbitrary positive real constant $\kappa$. Subharmonic fields $\boldsymbol{B}$ of that kind have been determined by 
numerical solution of the eigenvalue problem posed by the Fourier-transformed induction equation.

Instead of the cylindrical dynamo module we consider now a rectangular "dynamo box" with the edge lengths $L$ in the $x$ - and $y$-direction and $H$ in the $z$-direction. We consider a subharmonic field $\boldsymbol{B}$ such that the dynamo box contains just a "half wave" of its leading Fourier mode, that is, the mode with the largest period lengths. This means $N a / \sqrt{2}=L$ and $\kappa a / \sqrt{2}=H$. We then interpret the leading Fourier mode as the mean field $\overline{\boldsymbol{B}}$. Instead of characterizing the situation considered by the parameters $N$ and $\kappa$ we may also use the aspect ratios $L / H$ and $a / H$ of the dynamo box and of the spin generators.

Before presenting specific results derived from subharmonic solutions of the Roberts dynamo problem let us have a look on a result of the mean-field approach, that is, a solution of the Eq. (10) for $\bar{B}$ with $\alpha_{\perp}$ given by Eq. (20) which fits in the same sense to our dynamo box as we required it above for the leading mode of a subharmonic field $\boldsymbol{B}$. As can be easily shown the self-excitation condition reads

$R m_{\perp} R m_{\|} \phi\left(R m_{\perp}\right) \geq \frac{16 a}{\pi H}\left(1+2\left(\frac{H}{L}\right)^{2}\right)$

with $\phi$ as introduced with Eq. (20). We may rewrite this into

$R m_{\perp} R m_{\|}^{*} \phi\left(R m_{\perp}\right) \geq 1$

with $R m_{\|}^{*}$ defined by

$R m_{\|}=\frac{16 a}{\pi H}\left(1+2\left(\frac{H}{L}\right)^{2}\right) R m_{\|}^{*}$

Figure 18 shows the neutral line, $R m_{\perp} R m_{\|}^{*} \phi\left(R m_{\perp}\right)=1$, in the $R m_{\perp} R m_{\|}^{*}$-plane.

We return now to subharmonic fields $\boldsymbol{B}$ adjusted as described above to our dynamo box. Within this framework we rediscover the result Eq. (50), or Eq. (51), of the mean-field approach in the double limit $L / H \rightarrow \infty$ and $a / H \rightarrow 0$. Any deviation of $L / H$ and $a / H$ from this limit leads to higher requirements for dynamo action. In particular, if $R m_{\perp}$ is fixed, higher values of $R m_{\|}^{*}$ are necessary. For example, for $L / H \leq 2, a / H \rightarrow 0$ and $R m_{\perp} \leq 2$ the necessary values of $R m_{\|}^{*}$ are up to about $10 \%$ higher than predicted by the meanfield approach. Figure 18 shows the neutral line for $L / H=2$ in the $R m_{\perp} R m_{\|}^{*}$-plane obtained in the mean-field approach and three such lines derived from subharmonic solutions for finite $a / H$. Whereas in the mean-field approach dynamo action seems possible for arbitrary $R m_{\perp}$ if only $R m_{\|}^{*}$ is sufficiently large, we see now that it is only possible for not too small $R m_{\perp}$. That is, there is not only a critical value of $R m_{\|}^{*}$ but also a critical value of $R m_{\perp}$ so that a dynamo can never work without exceeding these values. If $a / H$ grows, for any given $R m_{\perp}$ the requirements to $R m_{\|}^{*}$ also grow.

Although the shape of our dynamo box is different from that of the real cylindrical dynamo module and the considered magnetic fields satisfy some kind of periodic boundary conditions rather than such which are realistic for this

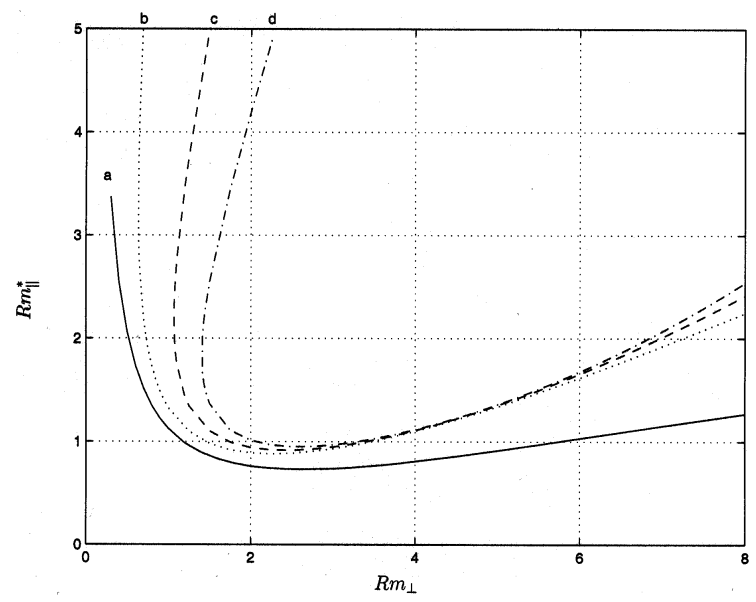

Fig. 18. Neutral lines for the rectangular dynamo box with $L / H=2$ in the $R m_{\perp} R m_{\|}^{*}$-plane. Line (a) is defined by the result $R m_{\perp} R m_{\|}^{*} \phi\left(R m_{\perp}\right)=1$ of the mean-field approach. The other lines are derived on the basis of subharmonic solutions for $L / H=2$ and various values of $a / H$, (b) for $a / H=0.177$, (c) for $a / H=0.283$ and (d) for $a / H=0.356$. The diagram essentially represents results shown in Fig. 6 of Plunian and Rädler (2002).

module, we may assume that the dependence of the selfexcitation condition on the aspect ratio $a / H$ for the real dynamo module is similar to that observed here. Considering that $L / H=2$ and $a / H=0.3$ correspond roughly to the real cylindrical dynamo module and that the dynamo works in a regime with $R m_{\perp}, R m_{\|}<2$, which implies $R m_{\|}^{*}<0.9$, we may conclude that the marginal value $C^{*}$ can again be up to $10 \%$ higher than predicted by the mean-field approach.

We may further conclude that the neutral line of the experimental device does not need to coincide exactly with an isoline of $C$ in the $V_{\mathrm{C}} V_{\mathrm{H}}$-diagram like Figs. 11 or 12 . Using the results represented in Fig. 18, expressing $R m_{\perp}$ and $R m_{\|}^{*}$ by $V_{\perp}$ and $V_{\|}$and putting as in the context of Eq. (21) again $V_{\perp}=V_{\mathrm{H}}$ and $V_{\|}=V_{\mathrm{C}}+V_{\mathrm{H}}$, we have constructed the neutral lines in $V_{\mathrm{C}} V_{\mathrm{H}}$-diagram. For reasons of comparability of these lines we have introduced $\tilde{V}_{\mathrm{C}}=\sqrt{H / a} V_{\mathrm{C}}$ and $\tilde{V}_{\mathrm{H}}=\sqrt{H / a} V_{\mathrm{H}}$. Figure 19 shows the neutral lines in a $\tilde{V}_{\mathrm{C}} \tilde{V}_{\mathrm{H}}$-diagram. The lines based on the subharmonic analysis with finite $a / H$ deviate from that obtained in the mean-field approach in the same sense as in Fig. 13 the experimentally determined neutral line deviates from the isolines of $C$ which were obtained in the mean-field approach. That is, this deviation is understandable as a consequence of the neglect of higher-order derivatives of $\overline{\boldsymbol{B}}$ in the mean-field approach.

7.4 A kinematic dynamo model with $\alpha_{\perp}$ varying across some boundary layer

Quite a few numerical investigations have been carried out with a kinematic dynamo model which deviates from those considered in Sect. 3 by assuming that $\alpha_{\perp}$ decays from its value in the interior of the dynamo module across some boundary layer to zero (Rädler et al., 1999). All induction 


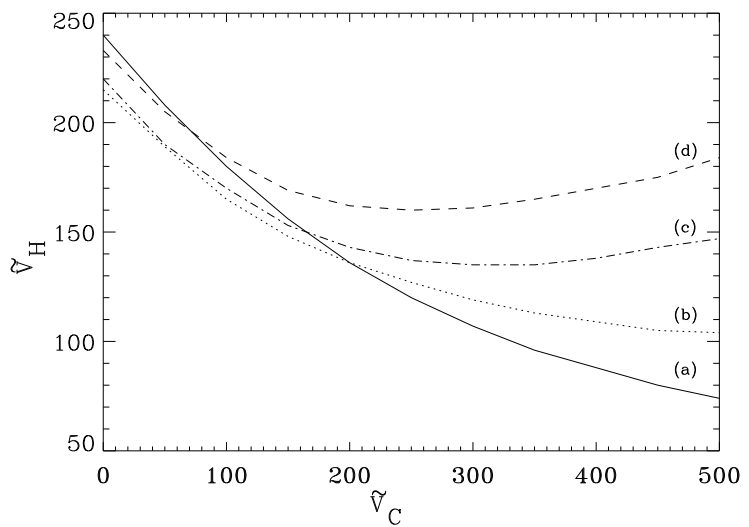

Fig. 19. Neutral lines for the rectangular dynamo box with $L / H=$

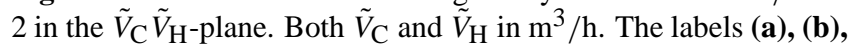
(c) and (d) correspond to those in Fig. 18.

Table 3. Marginal values $C^{*}$ for magnetic fields with different $m$. The lowest value of $C^{*}$ for $m=0$, which is given here, belongs to field of AS type.

\begin{tabular}{cccccc}
\hline$m$ & 0 & 1 & 2 & 3 & 4 \\
\hline$C^{*}$ & 8.432 & 7.276 & 9.262 & 11.35 & 13.54 \\
\hline
\end{tabular}

effects other than the $\alpha_{\perp}$-effect were again neglected.

In order to explain the distribution of $\alpha_{\perp}$ and $\sigma$, which was chosen with a view to the real structure of the dynamo module, we define first a small cylinder by $r \leq 0.941 R$ and $|z| \leq 0.458 H$, and a large cylinder by $r \leq 1.081 R$ and $|z| \leq 0.680 H$, where $r=\sqrt{x^{2}+y^{2}}$. We assume that $\alpha_{\perp}$ is constant inside the small cylinder, decreases in the space between the cylinders linearly in both $r$ and $z$ and vanishes on the surface of the large cylinder and outside it. Considering the large cylinder to be embedded in a sphere as shown in Fig. 3 we further assume that $\sigma$ is constant inside the large cylinder, is constant and smaller by a factor 100 in the remaining parts of the sphere and vanishes outside this sphere. We adopt the definition (12) of $C$ with $\alpha_{\perp}$ and $\eta$ interpreted as their values inside the small cylinder.

Marginal values $C^{*}$ of $C$ are given in Table 3 . The dynamo has again a non-oscillatory behaviour. The dependence of the growth rates $\lambda$ on $C$ is depicted in Fig. 20. Some aspects of the structure of the magnetic field with $m=1$ are shown in Fig. 21. The field is to a large extent concentrated inside the dynamo module and varies there strongly in $z$-direction.

\section{Concluding remarks}

The simple kinematic mean-field theory as explained in Sects. 2 to 5 describes indeed essential features of the Karlsruhe dynamo experiment. It predicts the structure of the most easily excitable magnetic field and the excitation condition in

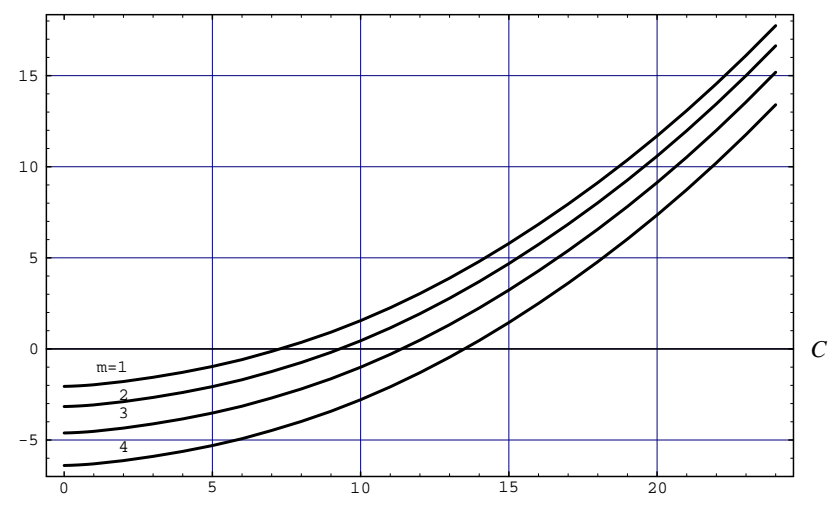

Fig. 20. The growth rates $\lambda$ in $\mathrm{s}^{-1}$ in dependence on $C$

its dependence on the rates of flow through the axial and the helical channels of the dynamo module. In agreement with these predictions magnetic fields were observed in the experiment which correspond to mean fields of the symmetry type $m=1$. As explained above it was clear from the very beginning that the marginal value $C^{*}$ of $C$, which defines the excitation condition, is somewhat underestimated by the simple theory. In Sects. 7.1 to 7.3 a few aspects are discussed which explain why the realistic value $C^{*}$ may well be up to $30 \%$ above the prediction of this theory. Considering these improvements of the theory there is again satisfactory agreement between experiment and theory. It seems even surprising that the experimentally determined region of $C^{*}$ is only about $10 \%$ above the prediction of the simple theory. There is a slight deviation of the predicted shape of the neutral line in the plane of the flow rates $V_{\mathrm{C}}$ and $V_{\mathrm{H}}$ through the two types of channels from the shape of the line derived from the measurements. Again this deviation is understandable with the corrections to the simple theory presented in Sect. 7.3. Of course the preferred orientation of the magnetic fields in the experimental device is, again by reasons already discussed, beyond the scope of the simple theory. In Sect. 6 we have studied the back-reaction of the magnetic field on the fluid motion, more precisely the pressure drop due to the magnetic field and its influence on the flow rates in the channels of the dynamo module, and developed on this basis a simple model for the dynamo in the nonlinear regime. In this way we gave estimates of the saturation field strengths of the dynamo, which are again in fair agreement with experimental findings.

\section{Appendix A Steady solution of Eq. (13) in the second- order approximation}

Consider Eq. (13), which apply for homogeneous fields $\overline{\boldsymbol{B}}$, in the steady case in the second-order approximation, that is,

$\eta \nabla^{2} \boldsymbol{B}^{\prime}=-(\overline{\boldsymbol{B}} \cdot \nabla) \boldsymbol{u}, \quad \nabla \cdot \boldsymbol{B}^{\prime}=0$.

We may put

$\boldsymbol{u}=\nabla \times \boldsymbol{a}, \quad \nabla \cdot \boldsymbol{a}=0$, 

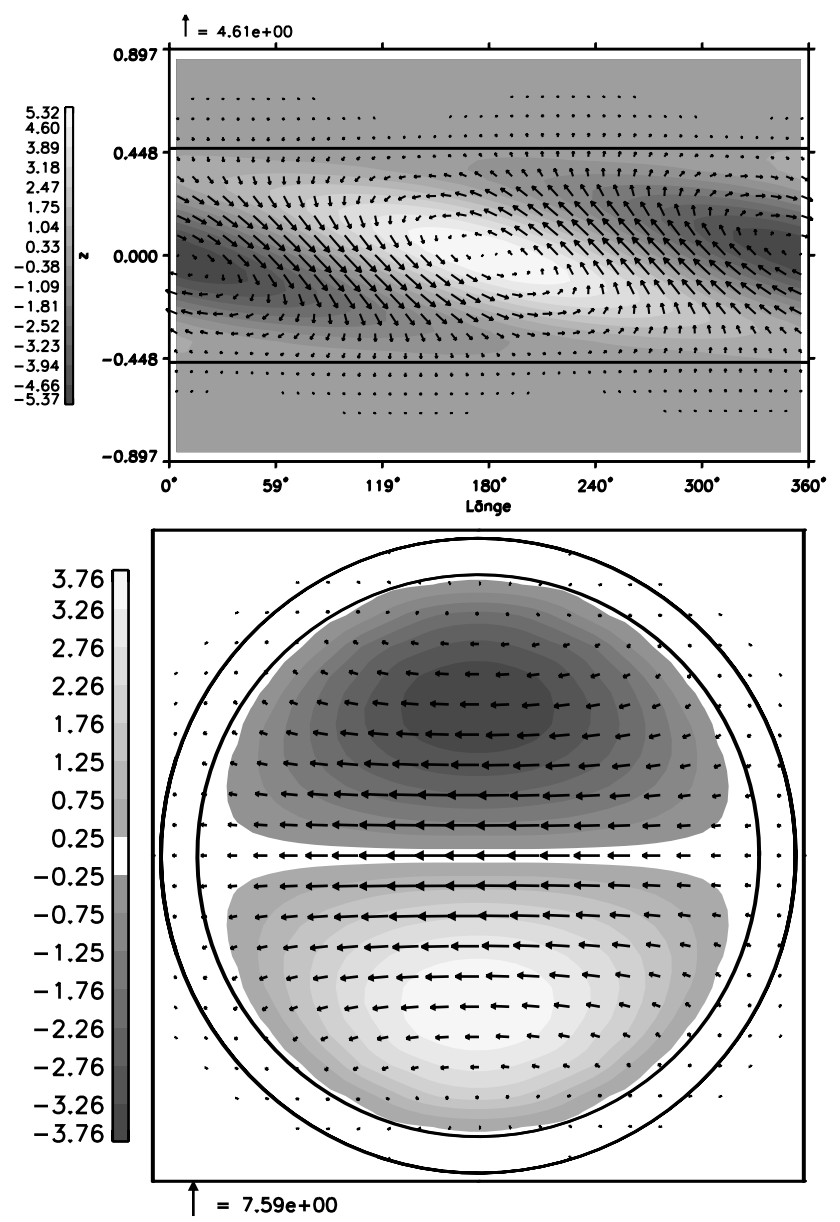

Fig. 21. Structure of the marginal magnetic field with $m=1$. Upper panel: cylindrical surface with radius $0.46 \mathrm{~m}$, lower panel: midplane. Vectors: components tangential to the surface, grey encoded: normal component

$\boldsymbol{a}=\nabla \times \tilde{\boldsymbol{a}}, \quad \nabla \cdot \tilde{\boldsymbol{a}}=0$,

so that

$\boldsymbol{u}=-\nabla^{2} \tilde{\boldsymbol{a}}$.

Then we have $\nabla^{2}\left(\eta \boldsymbol{B}^{\prime}-(\overline{\boldsymbol{B}} \cdot \nabla) \tilde{\boldsymbol{a}}\right)=\mathbf{0}$, that is $\eta \boldsymbol{B}^{\prime}-(\overline{\boldsymbol{B}} \cdot \nabla) \tilde{\boldsymbol{a}}=\nabla \Phi$ and $\Delta \Phi=0$, and can conclude that $\boldsymbol{B}^{\prime}=\frac{1}{\eta}(\overline{\boldsymbol{B}} \cdot \nabla) \tilde{\boldsymbol{a}}$.

In the case of the Roberts flow, in which $\boldsymbol{u}$ is given by Eq. (14) we have simply

$\tilde{\boldsymbol{a}}=\left(\frac{a}{\pi}\right)^{2} \boldsymbol{u}$.

For the spin generator flow with $\boldsymbol{u}$ defined by Eq. (22) inside the considered cell and being equal to zero outside we have

$\tilde{a}_{\varrho}=0$

$$
\tilde{a}_{\varphi}=\frac{\varrho}{2} \int_{\varrho}^{a / 2} u_{\varphi}\left(\varrho^{\prime}\right) d \varrho^{\prime}+\frac{1}{2 \varrho} \int_{0}^{\varrho} u_{\varphi}\left(\varrho^{\prime}\right) \varrho^{\prime 2} d \varrho^{\prime}
$$

Table A1. Some values of $\epsilon_{x}$ and $\epsilon_{y}$

\begin{tabular}{cccccccccc}
\hline$V_{\mathrm{H}}\left[\mathrm{m}^{3} / \mathrm{h}\right]$ & 0 & 25 & 50 & 75 & 100 & 125 & 150 & 175 & 200 \\
\hline$\epsilon_{x}$ & 0 & 0.17 & 0.30 & 0.39 & 0.45 & 0.47 & 0.48 & 0.48 & 0.47 \\
\hline$\epsilon_{y}$ & 0 & 0.21 & 0.44 & 0.68 & 0.91 & 1.13 & 1.31 & 1.47 & 1.61 \\
\hline \multicolumn{1}{c}{$\int^{a / 2} u_{z}\left(\varrho^{\prime}\right) \ln \left(\varrho^{\prime} / \varrho_{0}\right) \varrho^{\prime} d \varrho^{\prime}$} & & & & \\
$\tilde{a}_{z}=-$ & $\varrho$ \\
$-\ln (\varrho / \varrho 0)$ & $\int_{0} u_{z}\left(\varrho^{\prime}\right) \varrho^{\prime} d \varrho^{\prime}$.
\end{tabular}

Appendix B Concerning the determination of $\alpha_{\perp}$ for the spin generator flow in the second-order approximation

In contrast to our explanations on the second-order approximation in Sect. 4.3 we ignore here no longer the fluid motion outside the considered cell but assume again a flow pattern which is periodic everywhere. We continue to use, however, the cylindrical co-ordinate system $\varrho, \varphi, z$ with the axis $\varrho=0$ in the centre of a given cell and consider a Fourier decomposition of the fluid velocity $\boldsymbol{u}$ and of the magnetic fields $\overline{\boldsymbol{B}}$ and $\boldsymbol{B}^{\prime}$ with respect to $\varphi$, that is, a decomposition into modes proportional to $\exp (\operatorname{im} \varphi)$. As for $\boldsymbol{u}$ its part inside the given cell contributes only to modes with $m=0$, and that outside due to the symmetry of the flow pattern only to modes with $m= \pm 4, \pm 8, \cdots$. Since $\overline{\boldsymbol{B}}$ possesses only modes with $m= \pm 1$, the parts of $\boldsymbol{u}$ outside the given cell produce in the second-order approximation no other modes of $\boldsymbol{B}^{\prime}$ than such with $m= \pm 3, \pm 5, \pm 7, \cdots$. Thus these parts of $\boldsymbol{u}$ produce no contributions to the $\varrho, \varphi$ or $z$-components of $\boldsymbol{u} \times \boldsymbol{B}^{\prime}$ inside the given cell other than such with these $m$. Consequently its $x, y$ and $z$-components possess only contributions with $|m| \geq 2$. These vanish under averaging over this cell, that is, they do not contribute to $\overline{\boldsymbol{u} \times \boldsymbol{B}^{\prime}}$.

These considerations also make clear that in higher than second-order approximations the motion in neighbouring cells may well influence the average of $\boldsymbol{u} \times \boldsymbol{B}^{\prime}$ over the given cell.

\section{Appendix C Relations between local and mean magnetic fields on the axis of the dynamo module}

The magnetic probes on the axis of the dynamo module measure the components of the local magnetic field $\boldsymbol{B}$, which differs from the mean field $\overline{\boldsymbol{B}}$ by the fluctuations $\boldsymbol{B}^{\prime}$. According to the construction of the module the rotational motion of the fluid in the four spin-generators around the axis corresponds to flows away from $x=y=0$ in the vicinity of the $x$-axis 
and towards $x=y=0$ in the vicinity of the $y$-axis; see Fig. 2. Assuming that $\overline{\boldsymbol{B}}$ can be considered as a homogeneous field and using symmetry arguments we can conclude that

$$
B_{x}^{\prime}=-\epsilon_{x} \bar{B}_{x}, \quad B_{y}^{\prime}=\epsilon_{y} \bar{B}_{y}, \quad B_{z}^{\prime}=0
$$

or

$\bar{B}_{x}=\frac{B_{x}}{1-\epsilon_{x}}, \quad \bar{B}_{y}=\frac{B_{y}}{1+\epsilon_{y}}, \quad \bar{B}_{z}=B_{z}$,

at the axis of the dynamo module, with positive coefficients $\epsilon_{x}$ and $\epsilon_{y}$ depending on $V_{\mathrm{H}}$ but not on $V_{\mathrm{C}}$. These relations have been confirmed by numerical solutions of Eq. (13) for the spin generator flow. Some values of $\epsilon_{x}$ and $\epsilon_{y}$ obtained with these calculations are given in Table 4 . Note that $\boldsymbol{B}$ and $\overline{\boldsymbol{B}}$, and in particular their directions, can differ markedly.

Acknowledgements. The authors thank Prof. U. Müller for many helpful comments on the manuscript and Dr. M. Schüler for his assistance in preparing a number of figures of this paper.

\section{References}

Busse, F. H.: A model of the geodynamo, Geophys. J. R. Astron. Soc., 42, 437-459, 1975.

Busse, F. H.: Magnetohydrodynamics of the Earth's dynamo, Annu. Rev. Fluid Mech., 10, 435-462, 1978.

Busse, F. H.: Dynamo theory of planetary magnetism and laboratory experiments, in Evolution of Dynamical Structures in Complex Systems, edited by R. Friedrich and A. Wunderlin, vol. 69, pp. 197-207, Springer-Verlag Berlin, 1992.

Busse, F. H., Müller, U., Stieglitz, R., and Tilgner, A.: A two-scale homogeneous dynamo: An extended analytical model and an experimental demonstration under development, Magnetohydrodynamics, 32, 235-248, 1996.

Busse, F. H., Müller, U., Stieglitz, R., and Tilgner, A.: Spontaneous generation of magnetic fields in the laboratory, in Evolution of Spontaneous Structure in Dissipative Continuous Systems, edited by F. H. Busse and S. C. Müller, pp. 546 - 558, Springer, 1998.

Dobler, W. and Rädler, K.-H.: An integral equation approach to kinematic dynamo models, Geophys. Astrophys. Fluid Dyn., 89, $45-74,1998$.

Fuchs, H., Rädler, K.-H., and Schüler, M.: A numerical approach to dynamically consistent spherical dynamo models, in The Cosmic Dynamo, (Eds) Krause, F., Rädler, K.-H., and Rüdiger, G., pp. 129 - 133, Kluwer Academic Publishers Dordrecht Bosten London, 1993.

Gailitis, A.: Conditions of the self-excitation for a laboratory model of the geomagnetic dynamo, Magnitnaya Gidrodinamika, 1967, 45-54, in Russian, 1967.

Krause, F. and Rädler, K.-H.: Mean-Field Magnetohydrodynamics and Dynamo Theory, Akademie-Verlag Berlin and Pergamon Press Oxford, 1980.

Müller, U. and Stieglitz, R.: Can the Earth's magnetic field be simulated in the laboratory?, Naturwissenschaften, 87, 381-390, 2000

Müller, U. and Stieglitz, R.: The Karlsruhe dynamo experiment,
Nonlinear Processes in Geophysics, in print, 2002.

Plunian, F. and Rädler, K.-H., Subharmonic dynamo action in the Roberts flow, Geophys. Astrophys. Fluid Dyn., in print, 2002.

Rädler, K.-H. and Brandenburg, A.: Contributions to the theory of the Karlsruhe dynamo experiment, in preparation, 2002.

Rädler, K.-H., Apel, A., Apstein, E., and Rheinhardt, M.: Contributions to the theory of the planned Karlsruhe dynamo experiment, Tech. rep., Astrophysical Institute Potsdam, 1996.

Rädler, K.-H., Apstein, E., Rheinhardt, M., and Schüler, M.: Contributions to the theory of the planned Karlsruhe dynamo experiment - Supplements and Corrigenda, Tech. rep., Astrophysical Institute Potsdam, 1997a.

Rädler, K.-H., Apstein, E., and Schüler, M.: The alpha-effect in the Karlsruhe dynamo experiment, in Proceedings of the International Conference "Transfer Phenomena in Magnetohydrodynamic and Electroconducting Flows" held in Aussois, France, 1997, pp. 9-14, 1997b.

Rädler, K.-H., Apstein, E., Fuchs, H., and Rheinhardt, M.: Kurzbericht über Untersuchungen zum Projekt GEODYNAMO: Abschätzungen zu den Lorentz-Kräften. Zeitliche Entwicklung eines Magnetfeldes und maximale Feldstärke, Tech. rep., Astrophysikalisches Institut Potsdam, 1998a.

Rädler, K.-H., Apstein, E., Rheinhardt, M., and Schüler, M.: The Karlsruhe dynamo experiment - a mean-field approach, Studia geoph. et geod., 42, 224-231, 1998 b.

Rädler, K.-H., Apstein, E., and Rheinhardt, M.: Kurzbericht über Untersuchungen zum Projekt GEODYNAMO: Ein modifiziertes Modell des Dynamomoduls. Fremderregte Magnetfelder im unterkritischen Regime des Dynamos, Tech. rep., Astrophysikalisches Institut Potsdam, 1999.

Rädler, K.-H., Apstein, E., Fuchs, H., and Rheinhardt, M.: Kurzbericht über Untersuchungen zum Projekt GEODYNAMO: Studien zum Verhalten des Dynamos im nichtlinearen Regime auf der Grundlage eines einfachen Modelles. Abschätzungen zum Einfluss eines Magnetfeldes auf den Strömungsverlauf in den Spingeneratoren und den $\alpha$-Koeffizienten, Tech. rep., Astrophysikalisches Institut Potsdam, 2000a.

Rädler, K.-H., Apstein, E., Fuchs, H., and Rheinhardt, M.: Kurzbericht über Untersuchungen zum Projekt GEODYNAMO: Stabile und instabile stationäre Zustände des Dynamos im nichtlinearen Regime, Tech. rep., Astrophysikalisches Institut Potsdam, 2000b.

Roberts, G. O.: Dynamo action of fluid motions with twodimensional periodicity, Phil. Trans. Roy. Soc. London A, 271, 411-454, 1972.

Soward, A. M.: Fast dynamo action in a steady flow, J. Fluid Mech., 180, 267-295, 1987.

Stieglitz, R. and Müller, U.: GEODYNAMO - Eine Versuchsanlage zum Nachweis des homogenen Dynamoeffektes, Tech. rep., Forschungszentrum Karlsruhe für Technik und Umwelt, Wissenschaftliche Berichte FZKA 5716, 1996.

Stieglitz, R. and Müller, U.: Experimental demonstration of a homogeneous two-scale dynamo, Phys. Fluids, 13, 561-564, 2001.

Tilgner, A.: A kinematic dynamo with a small scale velocity field, Phys. Lett. A, 226, 75-79, 1996.

Tilgner, A.: Predictions on the behavior of the Karlsruhe dynamo, Acta Astron. et Geophys. Univ. Comenianae, 19, 51-62, 1997.

Tilgner, A. and Busse, F.: Subharmonic dynamo action of fluid motions with two-dimensional periodicity, Proc. R. Soc. London, Ser. A, 448, 237-244, 1995. 